\title{
Targeting EGFR Tyrosine Kinase: Design, Synthesis and Biological Evaluation of Novel Quinazolinone Derivatives
}

\author{
Manijeh Nematpour ${ }^{1}$, Elham Rezaee (id ${ }^{1,{ }^{*}}$, Maryam Nazari (iD ${ }^{1}$, Omid Hosseini ${ }^{2}$ and Sayyed Abbas \\ Tabatabai (iD ${ }^{1, * *}$ \\ ${ }^{1}$ Department of Pharmaceutical Chemistry, School of Pharmacy, Shahid Beheshti University of Medical Sciences, Tehran, Iran \\ ${ }^{2}$ Central Research Labretories, Shahid Beheshti University of Medical Sciences, Tehran, Iran \\ "Corresponding author: Department of Pharmaceutical Chemistry, School of Pharmacy, Shahid Beheshti University of Medical Sciences, Tehran, Iran. Email: \\ elham_rezaee63@yahoo.com \\ "Corresponding author: Department of Pharmaceutical Chemistry, School of Pharmacy, Shahid Beheshti University of Medical Sciences, Tehran, Iran. Email: \\ sa_tabatabai@yahoo.com
}

Received 2021 October 16; Revised 2022 January 03; Accepted 2022 January 03.

\begin{abstract}
Impaired cell cycle regulation and disturbance in signal transduction pathway are two major causes of a condition defined as cancer, one of the significant reasons for mortality worldwide. Epidermal growth factor receptor tyrosine kinase inhibitors (EGFR-TKIs) have been commonly used as anticancer agents, and the majority of this medications possess quinazoline moiety as a heteroaromatic core. In this study, two novel series of EGFR-TKIs containing quinazolinone core were designed and synthesized. Most compounds showed reasonable inhibitory activity against EGFR-TK compared to that of erlotinib, a reversible inhibitor of this enzyme. Compound 8b, 2-((2-chlorobenzyl)amino)-6-phenoxyquinazolin-4(1H)-one, with an $\mathrm{IC}_{50}$ value of $1.37 \mathrm{nM}$ exhibited the highest potency. Molecular docking study of compound $8 \mathrm{~b}$ showed that it had the same direction of erlotinib and formed proper hydrogen bonds and hydrophobic interactions with the important amino acid residues of the active site. Based on in-silico calculations of ADME properties, our novel compounds have the potential to be orally active agents.
\end{abstract}

Keywords: Anticancer, Biological Activity, EGFR, Quinazolinone, Tyrosine Kinase

\section{Background}

Nowadays, cancer is one of the leading causes of death worldwide, and discovering new anticancer agents with high selectivity and low adverse effects is a significant challenge encountered by researchers $(1,2)$. Receptor protein tyrosine kinases (RPTKs), also called phosphotyrosine kinases, have a considerable role in the signal transduction pathway and are responsible for regulating various functions in normal cells, including cell growth, proliferation, metabolism, differentiation, and apoptosis $(3,4)$. The alteration of this enzyme via mutations would lead to dysregulated cell cycle and uncontrolled cell duplication, defined as cancer (5-7). The FDA approves 47 kinase inhibitors over the last 30 years that have developed as anticancer agents (8).

The human epidermal growth factor receptors (EGFRs) belong to a class of RPTKs, including four members: EGFR/ERBB1/HER1, ERBB2/HER2, ERBB3/HER3 and ERBB4/HER4 (9). This type of receptors can perform a key role in signal transduction for adjusting cell-growth and developing many types of solid tumors, including head and neck, lung, breast, bladder, prostate, and kidney cancers (10-12).

As a relatively lethal disease, lung cancer's manifestations are categorized into two main groups: small-cell lung carcinoma (SCLC) and non-small-cell lung carcinoma (NSCLC) $(13,14)$. This invasive and metastatic cancer type and stage would guide the specialist to select proper treatment, including surgery, radiation, and chemotherapy. Erlotinib (Tarceva®), an EGFR tyrosine kinase inhibitor (EGFR-TKI), has been approved as the first-line therapy for EGFR mutated progressive or metastatic NSCLC (15). Quinazolines are ubiquitous heterocyclic scaffolds found in various pharmaceuticals and natural products (16). The quinazoline-containing derivatives have been contributed to the variety of EGFR-TKIs. There have been many novel quinazoline derivatives possessing high inhibitory activity compared to FDA approved drugs in the last few years. Some novel and potent EGFR-TKIs with quinazoline moiety were illustrated in Figure 1. Compound 1 is a semicarbazone fused quinazoline group derivative with an 


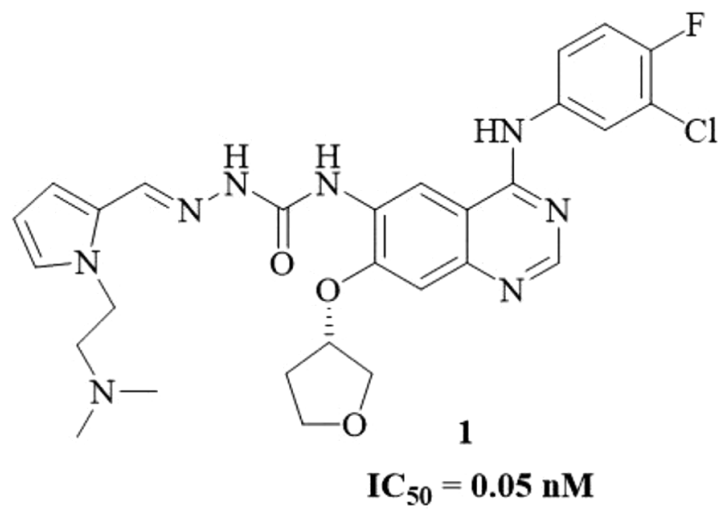<smiles>COCCCOc1cc2ncnc(Nc3ccc(F)c(Cl)c3)c2cc1NC(=O)/C=C/CN(C)C</smiles>

$$
\mathrm{IC}_{50}=0.76 \mathrm{nM}
$$<smiles>Cc1cc2ncnc(N3CCc4ccccc43)c2cc1NC(=O)/C=C/CN(C)C</smiles>

3

$\mathrm{IC}_{50}=3 \mathrm{nM}$<smiles>COc1cc2c(Nc3ccc(F)c(Cl)c3)ncnc2cc1OCCCN1C[C@H](O)[C@@H](O)[C@H](O)C1CO</smiles>

$\mathrm{IC}_{50}=1.79 \mathrm{nM}$

Figure 1. The structures of some potent EGFR-TKIs containing quinazoline moiety.

$\mathrm{IC}_{50}$ value of $0.05 \mathrm{nM}(17)$. The concept behind the designing compound $2\left(\mathrm{IC}_{50}=0.76 \mathrm{nM}\right)$ was changing the size of alkoxyalkane chains at 7-position of quinazoline core (18). Compound $3\left(\mathrm{IC}_{50}=3 \mathrm{nM}\right.$ ) bearing 2,3-dihydroindolequinoline core demonstrated strong potency as well as excellent antiproliferative activity (19). Compound $4\left(\mathrm{IC}_{50}=1.79 \mathrm{nM}\right)$ was designed as dual EGFR and $\alpha$ glucosidase inhibitor possessing quinazoline moiety hybridized with 1-deoxynojirimycin segment (20).

Based on studies, drug resistance in cancer and ineffective chemotherapy mainly stem from EGFR mutation (replacement of the threonine 790 with methionine 790), leading to the progression of cancer stages. Although this factor could be conquered through developing inhibitors binding covalently to the ATP binding site of EGFR (21), these inhibitors suffer from weak selectivity due to their affinity to other kinases (22). The alternative strategy introduces reversible EGFR-TKIs, which have been desirable recently, and researchers are working on them.

Considering the necessary features of erlotinib as a reversible inhibitor and related structures, we designed two series of novel quinazolinone derivatives as EGFR-TKIs il- lustrated in Figure 2. According to the essential pharmacophoric features of erlotinib (3), a hydrophobic head and tail on a heteroaromatic system and a hydrogen acceptor spacer that bridges between head and heteroaromatic system are required to locate a compound into the active site of EGFR tyrosine kinase (EGFR-TK). Therefore, the modifications of our novel series of quinazolinone derivatives (compounds 8 and 9) included the introduction of various N-aryl and -alkyl groups (hydrophobic head) with different substituents at the 2-position of quinazolinone moiety (bioisoster of quinazoline group) and the amino group served as a spacer. Besides, hydrogen at the 6-position was replaced with phenoxy group in 8a-o derivatives to resemble a hydrophobic tail (Figure 2). Finally, the activities of the novel compounds toward recombinant EGFR-TK were evaluated.

\section{Methods}

\subsection{Chemistry}

All reagents and solvents were purchased from Aldrich or Merck Company and utilized without any purification. 


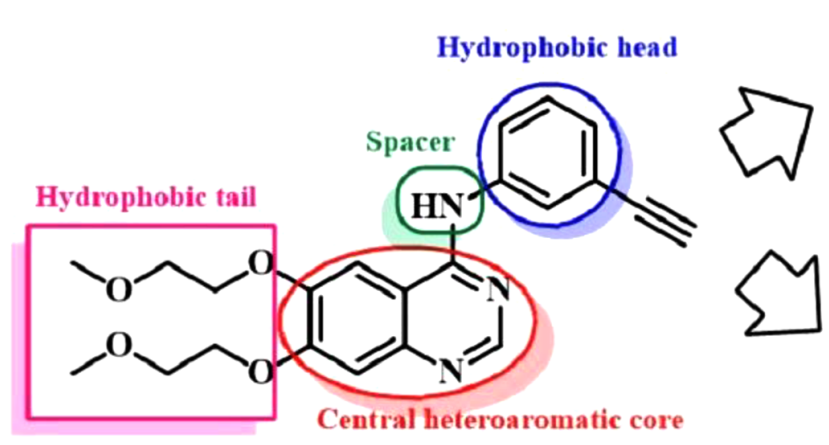

Erlotinib
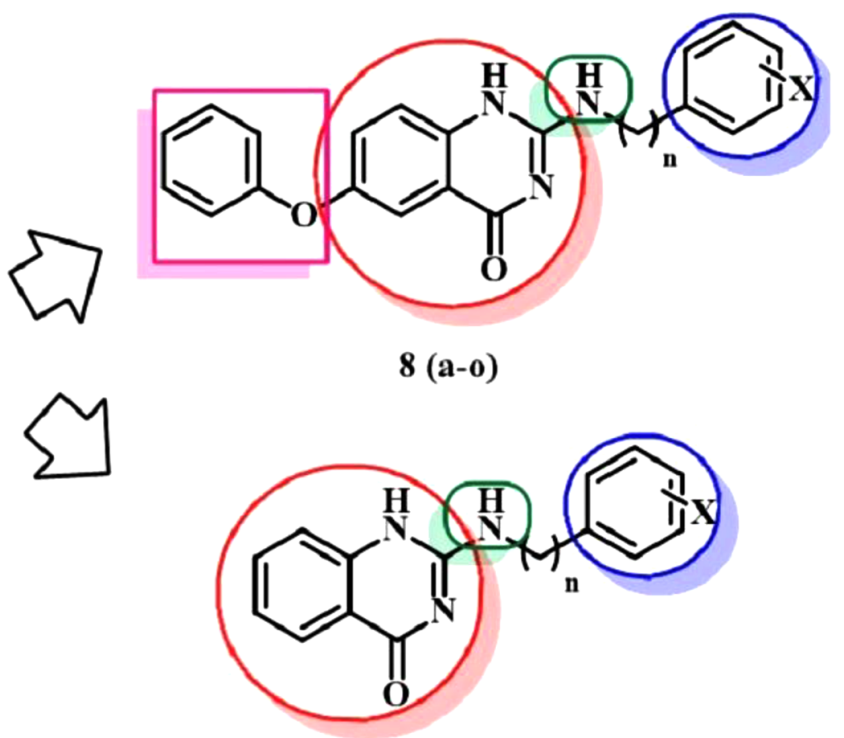

9 (a-0)

Figure 2. The design strategy of our novel compounds as EGFR-TKIs based on pharmacophoric features of erlotinib.

IR spectra were measured on a Perkin Elmer 834 spectrometer. The absorptions were reported on the wavenumber $\left(\mathrm{cm}^{-1}\right)$ scale, in the range $400-4000 \mathrm{~cm}^{-1}$. All the ${ }^{1} \mathrm{H}-\mathrm{NMR}$ and ${ }^{13} \mathrm{C}$-NMR spectra were recorded on a Bruker Avance II spectrophotometer using $\mathrm{CDCl}_{3}$ as a solvent and tetramethylsilane as an internal standard. Finnigan-MAT-8430EIMS was applied to obtain mass spectra; at $70 \mathrm{eV}$; in $\mathrm{m} / \mathrm{z}$ (rel.\%). Melting points were obtained using Electrothermal 9100 apparatus and were uncorrected.

\subsection{General Procedure for the Synthesis of Compounds 8a-o}

A mixture of 2-amino-5-fluorobenzoic acid (5.0 mmol), phenol (5.2 mmol), CuI (0.1 mmol), NaH (2.0 mmol) in DMF (3 mL) was refluxed at $110^{\circ} \mathrm{C}$ for $2 \mathrm{~h}$. After the addition of ethyl chloroformate $(6.0 \mathrm{mmol})$ to the medium, $\mathrm{Et}_{3} \mathrm{~N}(2.0$ $\mathrm{mmol}$ ) was added dropwise over $10 \mathrm{~min}$ at room temperature and refluxed at $110^{\circ} \mathrm{C}$ for $3 \mathrm{~h}$. Then, the mixture was cooled, diluted with $\mathrm{CH}_{2} \mathrm{Cl}_{2}(2 \mathrm{~mL})$ and aqueous $\mathrm{NH}_{4} \mathrm{Cl}$ solution (3 mL), and stirred for $30 \mathrm{~min}$. The aqueous layer was extracted with $\mathrm{CH}_{2} \mathrm{Cl}_{2}$, dried over sodium sulfate anhydrous, and concentrated under reduced pressure. The precipitate was recrystallized from EtOH $(30 \mathrm{~mL})$ to afford 6-phenoxyisatoic anhydride.

To a mixture of appropriate aniline or benzylamine (3.0 mmol) and trichloroacetonitrile $(3.5 \mathrm{mmol})$, a mixture of synthesized 6-phenoxyisatoic anhydride (3.5 mmol) and
$\mathrm{CuO}(0.1 \mathrm{mmol})$ in DMF $(3 \mathrm{~mL})$ was slowly added and refluxed at $110^{\circ} \mathrm{C}$ for $4 \mathrm{~h}$. The end of the reaction was controlled by TLC (eluent: AcOEt/ hexane 1:3). Then, the mixture was diluted with $\mathrm{CH}_{2} \mathrm{Cl}_{2}(2 \mathrm{~mL})$ and aqueous $\mathrm{NH}_{4} \mathrm{Cl}$ solution ( $3 \mathrm{~mL}$ ) and stirred for $30 \mathrm{~min}$. The aqueous layer was extracted with $\mathrm{CH}_{2} \mathrm{Cl}_{2}$ dried over sodium sulfate anhydrous and concentrated under reduced pressure. The precipitate was washed with diethyl ether to afford final product 8.

\subsubsection{2-(Benzylamino)-6-phenoxyquinazolin-4(1H)-one (8a)}

White powder; yield: $0.30 \mathrm{~g}$ (88\%); mp: $199-201^{\circ} \mathrm{C}$. IR $(\mathrm{KBr})\left(\nu_{\max }, \mathrm{cm}^{-1}\right): 3201,3101,1651,1644,1124,1100 .{ }^{1} \mathrm{H}-\mathrm{NMR}$ $\left(500 \mathrm{MHz}, \mathrm{CDCl}_{3}\right): \delta_{\mathrm{H}}=4.02\left(2 \mathrm{H}, \mathrm{s}, \mathrm{CH}_{2}\right), 6.08(1 \mathrm{H}, \mathrm{s}, \mathrm{NH})$, 7.29 - 7.36 ( $7 \mathrm{H}, \mathrm{m}, \mathrm{H}_{3,4,5}$-phenoxy, $\mathrm{H}_{5}$-quinazolinone, $\mathrm{H}_{3,4,5}$ benzylamine), $7.40\left(1 \mathrm{H}, \mathrm{d},{ }^{3} \mathrm{~J}=7.9 \mathrm{~Hz}, \mathrm{H}_{7}\right.$-quinazolinone), $7.51\left(1 \mathrm{H}, \mathrm{d},{ }^{3} \mathrm{~J}=7.9 \mathrm{~Hz}, \mathrm{H}_{8}\right.$-quinazolinone), $7.78(2 \mathrm{H}, \mathrm{d}$, ${ }^{3} \mathrm{~J}=7.5 \mathrm{~Hz}, \mathrm{H}_{2,6}$-phenoxy), $7.93\left(2 \mathrm{H}, \mathrm{d},{ }^{3} \mathrm{~J}=7.8 \mathrm{~Hz}, \mathrm{H}_{2,6}\right.$ benzylamine), $9.97(1 \mathrm{H}, \mathrm{s}, \mathrm{NH}) \cdot{ }^{13} \mathrm{C}-\mathrm{NMR}\left(125.7 \mathrm{MHz}, \mathrm{CDCl}_{3}\right)$ : $45.9\left(\mathrm{CH}_{2}\right), 127.4(2 \mathrm{CH}), 128.7(2 \mathrm{CH}), 129.3(\mathrm{CH}), 130.1(2 \mathrm{CH})$, $130.7(2 \mathrm{CH}), 132.5(\mathrm{CH}), 132.9(\mathrm{CH}), 133.0(2 \mathrm{CH}), 134.6(\mathrm{C}), 135.7$ (C), 142.1(C), 146.0 (C), 147.3(C), 166.2 (C), 176.0 (C). EI-MS:343 ( $\left.\mathrm{M}^{+}, 14\right), 266$ (58), 250 (20), 238 (77), 211 (59), 133 (100), 106 (44), 93 (55). 
2.2.2. 2-((2-Chlorobenzyl)amino)-6-phenoxyquinazolin-4(1H)one $(8 b)$

White powder; yield: $0.34 \mathrm{~g}$ (89\%); mp: $234-236^{\circ} \mathrm{C}$. IR $(\mathrm{KBr})\left(\nu_{\mathrm{max}}, \mathrm{cm}^{-1}\right): 3458,3324,1658,1625,1254,1010 .{ }^{1} \mathrm{H}-\mathrm{NMR}$ $\left(500 \mathrm{MHz}, \mathrm{CDCl}_{3}\right): \delta_{\mathrm{H}}=4.45\left(2 \mathrm{H}, \mathrm{s}, \mathrm{CH}_{2}\right), 6.01(1 \mathrm{H}, \mathrm{s}, \mathrm{NH})$, $7.36\left(1 \mathrm{H}, \mathrm{t},{ }^{3} \mathrm{~J}=7.9 \mathrm{~Hz}, \mathrm{H}_{4}\right.$-phenoxy), 7.47 - $7.51\left(7 \mathrm{H}, \mathrm{m}, \mathrm{H}_{2,3,5,6^{-}}\right.$ phenoxy, $\mathrm{H}_{5,7}$-quinazolinone, H5-benzylamine), $7.60(1 \mathrm{H}$, $\mathrm{t},{ }^{3} \mathrm{~J}=7.9 \mathrm{~Hz}, \mathrm{H}_{4}$-benzylamine), $7.91\left(1 \mathrm{H}, \mathrm{d},{ }^{3} \mathrm{~J}=7.9 \mathrm{~Hz}, \mathrm{H}_{8}\right.$ quinazolinone), 7.97 ( $2 \mathrm{H}, \mathrm{d},{ }^{3} \mathrm{~J}=7.5 \mathrm{~Hz}, \mathrm{H} 3,6$ - benzylamine), 9.99 (1 H, s, NH). ${ }^{13} \mathrm{C}-\mathrm{NMR}\left(125.7 \mathrm{MHz}, \mathrm{CDCl}_{3}\right): 46.6\left(\mathrm{CH}_{2}\right), 127.5$ (CH), 128.8 (2 CH), $129.0(\mathrm{CH}), 129.2(\mathrm{CH}), 129.3(\mathrm{CH}), 130.7$ $(\mathrm{CH}), 131.6(2 \mathrm{CH}), 131.8(2 \mathrm{CH}), 131.9(\mathrm{CH}), 132.0(\mathrm{C}), 134.4(\mathrm{C})$, 134.5 (C), 140.3 (C), 142.1 (C), 147.2 (C), 161.2 (C), 172.7 (C). EIMS: 377 (M+ $\mathrm{M}^{+}$), 266 (19), 252 (35), 238 (29), 211 (60), 168 (55), 111 (100), 93 (52).

2.2.3. 2-((4-Methoxybenzyl)amino)-6-phenoxyquinazolin$4(1 \mathrm{H})$-one ( $8 \mathrm{c})$

White powder; yield: $0.30 \mathrm{~g}$ (81\%); mp: $245-247^{\circ} \mathrm{C}$. IR $(\mathrm{KBr})\left(\nu_{\max }, \mathrm{cm}^{-1}\right): 3421,3320,1651,1620,1204,1000 .{ }^{1} \mathrm{H}-$ NMR $\left(500 \mathrm{MHz}, \mathrm{CDCl}_{3}\right): \delta_{\mathrm{H}}=3.36\left(3 \mathrm{H}, \mathrm{s}, \mathrm{OCH}_{3}\right), 4.45(2 \mathrm{H}$, s, $\mathrm{CH}_{2}$ ), $6.11(1 \mathrm{H}, \mathrm{s}, \mathrm{NH}), 7.28$ - 7.36 ( $5 \mathrm{H}, \mathrm{m}, \mathrm{H}_{2,6}$-phenoxy, $\mathrm{H}_{7}$ quinazolinone, $\mathrm{H}_{3,5}$-benzylamine), 7.47-7.50 (4 H, m, $\mathrm{H}_{3,5^{-}}$ phenoxy, $\mathrm{H}_{5,8}$-quinazolinone), $7.61\left(1 \mathrm{H}, \mathrm{t},{ }^{3} \mathrm{~J}=7.9 \mathrm{~Hz}, \mathrm{H}_{4}\right.$ phenoxy), 7.96 ( $2 \mathrm{H}, \mathrm{d},{ }^{3} \mathrm{~J}=7.8 \mathrm{~Hz}, \mathrm{H}_{2,6}$-benzylamine), 9.00 $(1 \mathrm{H}, \mathrm{s}, \mathrm{NH}) .{ }^{13} \mathrm{C}-\mathrm{NMR}\left(125.7 \mathrm{MHz}, \mathrm{CDCl}_{3}\right): 47.5\left(\mathrm{CH}_{2}\right), 58.2$ $\left(\mathrm{OCH}_{3}\right), 128.8(\mathrm{CH}), 129.2(2 \mathrm{CH}), 129.4(2 \mathrm{CH}), 132.5(2 \mathrm{CH})$, $133.2(\mathrm{CH}), 133.3(\mathrm{CH}), 134.1(\mathrm{C}), 136.1(2 \mathrm{CH}), 137.6(\mathrm{CH}), 138.1$ (C), $139.7(\mathrm{C}), 140.0(\mathrm{C}), 145.2(\mathrm{C}), 150.3(\mathrm{C}), 161.2(\mathrm{C}), 173.3(\mathrm{C})$. EI-MS: $373\left(\mathrm{M}^{+}, 12\right), 266$ (24), 252 (44), 136 (39), 122 (44), 107 (100), 93 (55).

2.2.4. 2-((4-Chlorobenzyl)amino)-6-phenoxyquinazolin-4(1H)one $(8 d)$

Gray powder; yield: $0.31 \mathrm{~g}(83 \%)$; mp: $251-253^{\circ} \mathrm{C}$. IR $(\mathrm{KBr})\left(\nu_{\max }, \mathrm{cm}^{-1}\right): 3524,3399,1693,1679,1254,1025 .{ }^{1} \mathrm{H}-$ $\operatorname{NMR}\left(500 \mathrm{MHz}, \mathrm{CDCl}_{3}\right): \delta_{\mathrm{H}}=4.06\left(2 \mathrm{H}, \mathrm{s}, \mathrm{CH}_{2}\right), 6.13(1 \mathrm{H}, \mathrm{s}$, $\mathrm{NH}$ ), 7.26 - 7.31 (5 H, m, $\mathrm{H}_{2,6}$-phenoxy, $\mathrm{H}_{3,5}$-benzylamine, $\mathrm{H}_{5}$ quinazolinone), $7.44\left(2 \mathrm{H}, \mathrm{d},{ }^{3} \mathrm{~J}=7.9 \mathrm{~Hz}, \mathrm{H}_{7,8}\right.$-quinazolinone), $7.57\left(1 \mathrm{H}, \mathrm{t},{ }^{3} \mathrm{~J}=7.9 \mathrm{~Hz}, \mathrm{H}_{4}\right.$-phenoxy), $7.69\left(2 \mathrm{H}, \mathrm{t},{ }^{3} \mathrm{~J}=7.9 \mathrm{~Hz}\right.$, $\mathrm{H}_{3,5}$-phenoxy), 7.87 (2 H, d, ${ }^{3} \mathrm{~J}=7.8 \mathrm{~Hz}, \mathrm{H}_{2,6}$-benzylamine), 8.06 (1 H, s, NH). ${ }^{13} \mathrm{C}-\mathrm{NMR}\left(125.7 \mathrm{MHz}, \mathrm{CDCl}_{3}\right): 47.6\left(\mathrm{CH}_{2}\right), 123.5$ (2 CH), $127.4(\mathrm{CH}), 128.8(\mathrm{CH}), 129.3(2 \mathrm{CH}), 130.2(2 \mathrm{CH}), 132.5$ $(\mathrm{CH}), 133.1(\mathrm{C}), 135.7(2 \mathrm{CH}), 136.6(\mathrm{CH}), 137.0(\mathrm{C}), 139.9(\mathrm{C}), 144.7$ (C), 146.2 (C), 151.3 (C), 161.3 (C), 167.0 (C). EI-MS: 379 (M+2, 6), 377 (12), 284 (24), 266 (29), 252 (40), 111 (100), 93 (70).

2.2.5. 2-((4-Fluorobenzyl)amino)-6-phenoxyquinazolin-4(1H)one (8e)

White powder; yield: $0.28 \mathrm{~g}$ (77\%); mp: $220-222^{\circ} \mathrm{C}$. IR $(\mathrm{KBr})\left(\nu_{\mathrm{max}}, \mathrm{cm}^{-1}\right): 3449,3306,1690,1672,1258,1024 .{ }^{1} \mathrm{H}-\mathrm{NMR}$ $\left(500 \mathrm{MHz}, \mathrm{CDCl}_{3}\right): \delta_{\mathrm{H}}=4.13\left(2 \mathrm{H}, \mathrm{s}, \mathrm{CH}_{2}\right), 6.23(1 \mathrm{H}, \mathrm{s}, \mathrm{NH})$, 7.29 - 7.33 ( $6 \mathrm{H}, \mathrm{m}, \mathrm{H}_{2,4,6}$-phenoxy, $\mathrm{H}_{5}$-quinazolinone, $\mathrm{H}_{3,5}$ benzylamine), $7.40\left(1 \mathrm{H}, \mathrm{d},{ }^{3} \mathrm{~J}=7.9 \mathrm{~Hz}, \mathrm{H}_{7}\right.$-quinazolinone), 7.48 - 7.51 (4 H, m, $\mathrm{H}_{2,6}$-benzylamine, $\mathrm{H}_{3,5}$-phenoxy), 7.92 (1 $\mathrm{H}, \mathrm{d},{ }^{3} \mathrm{~J}=7.8 \mathrm{~Hz}, \mathrm{H}_{8}$-quinazolinone), $8.73(1 \mathrm{H}, \mathrm{s}, \mathrm{NH}) .{ }^{13} \mathrm{C}$-NMR (125.7MHz, $\left.\mathrm{CDCl}_{3}\right)$ : $47.2\left(\mathrm{CH}_{2}\right), 122.5(2 \mathrm{CH}), 127.5(2 \mathrm{CH}), 128.7$ (CH), $129.2(\mathrm{CH}), 130.7(2 \mathrm{CH}), 132.5(\mathrm{CH}), 133.0(2 \mathrm{CH}), 134.7$ (CH), 135.6(C), 136.6 (C), 137.7(C), 142.1(C), 144.5(C), 147.3(C), 161.2 (C), 169.0 (C). EI-MS: $361\left(\mathrm{M}^{+}, 9\right), 266$ (15), 252 (24), 238 (25), 124 (36), 110 (100), 95 (85), 93 (41).

2.2.6. 2-((4-Methylbenzyl)amino)-6-phenoxyquinazolin-4(1H)one (8f)

White powder; yield: $0.26 \mathrm{~g}$ (72\%); mp: $229-231^{\circ} \mathrm{C}$. IR $(\mathrm{KBr})\left(\nu_{\max }, \mathrm{cm}^{-1}\right): 3455,3325,1688,1654,1287,1021 .{ }^{1} \mathrm{H}-\mathrm{NMR}$ $\left(500 \mathrm{MHz}, \mathrm{CDCl}_{3}\right): \delta_{\mathrm{H}}=2.66\left(3 \mathrm{H}, \mathrm{s}, \mathrm{CH}_{3}\right), 4.46\left(2 \mathrm{H}, \mathrm{s}, \mathrm{CH}_{2}\right)$, $6.19(1 \mathrm{H}, \mathrm{s}, \mathrm{NH}), 7.21\left(1 \mathrm{H}, \mathrm{d},{ }^{3} \mathrm{~J}=7.8 \mathrm{~Hz}, \mathrm{H}_{7}\right.$-quinazolinone), 7.22 - 7.26 ( $6 \mathrm{H}, \mathrm{m}, \mathrm{H}_{3,4,5}$-phenoxy, $\mathrm{H}_{5}$-quinazolinone, $\mathrm{H}_{3,5}$ benzylamine), 7.33 ( $2 \mathrm{H}, \mathrm{d},{ }^{3} \mathrm{~J}=7.5 \mathrm{~Hz}, \mathrm{H}_{2,6}$-phenoxy), 7.35 $\left(1 \mathrm{H}, \mathrm{d},{ }^{3} \mathrm{~J}=7.8 \mathrm{~Hz}, \mathrm{H}_{8}\right.$-quinazolinone), $7.91\left(2 \mathrm{H}, \mathrm{d},{ }^{3} \mathrm{~J}=7.5\right.$ $\mathrm{Hz}, \mathrm{H}_{2,6}$-benzylamine), $9.02(1 \mathrm{H}, \mathrm{s}, \mathrm{NH}) .{ }^{13} \mathrm{C}-\mathrm{NMR}(125.7 \mathrm{MHz}$, $\left.\mathrm{CDCl}_{3}\right): 22.2\left(\mathrm{CH}_{3}\right), 45.7\left(\mathrm{CH}_{2}\right), 126.2(\mathrm{C}), 127.5(2 \mathrm{CH}), 127.7$ $(\mathrm{CH}), 130.0(\mathrm{CH}), 130.6(\mathrm{CH}), 131.3(2 \mathrm{CH}), 132.2(2 \mathrm{CH}), 133.0$ (CH), $134.0(2 \mathrm{CH}), 134.9(\mathrm{CH}), 137.6(\mathrm{C}), 138.8(\mathrm{C}), 140.6(\mathrm{C})$, 143.4 (C), 161.3 (C), $171.1(\mathrm{C})$. EI-MS: $357\left(\mathrm{M}^{+}, 15\right), 266$ (19), 252 (21), 238 (100), 120 (54), 93 (88).

\subsubsection{6-Phenoxy-2-(phenylamino)quinazolin-4(1H)-one (8g)}

White powder; yield: $0.24 \mathrm{~g}$ (74\%); mp: $214-216^{\circ} \mathrm{C}$. IR $(\mathrm{KBr})\left(\nu_{\max }, \mathrm{cm}^{-1}\right): 3465,3385,1681,1657,1285,1064 .{ }^{1} \mathrm{H}-\mathrm{NMR}$ $\left(500 \mathrm{MHz}, \mathrm{CDCl}_{3}\right): \delta_{\mathrm{H}}=6.22(1 \mathrm{H}, \mathrm{s}, \mathrm{NH}), 7.30-7.36(8 \mathrm{H}, \mathrm{m}$, $\mathrm{H}_{3,4,5}$-phenyl, $\mathrm{H}_{3,4,5}$-phenoxy, $\mathrm{H}_{5,7}$-quinazolinone), $7.40(1 \mathrm{H}$, $\mathrm{d},{ }^{3} \mathrm{~J}=7.8 \mathrm{~Hz}, \mathrm{H}_{8}$-quinazolinone $), 7.50\left(2 \mathrm{H}, \mathrm{d},{ }^{3} \mathrm{~J}=7.5 \mathrm{~Hz}, \mathrm{H}_{2,6^{-}}\right.$ phenoxy), $7.93\left(2 \mathrm{H}, \mathrm{d},{ }^{3} \mathrm{~J}=7.9 \mathrm{~Hz}, \mathrm{H}_{2,6}\right.$-phenyl), $8.12(1 \mathrm{H}, \mathrm{s}$, $\mathrm{NH}) .{ }^{13} \mathrm{C}-\mathrm{NMR}\left(125.7 \mathrm{MHz}, \mathrm{CDCl}_{3}\right): 126.7(2 \mathrm{CH}), 127.3(2 \mathrm{CH})$, $127.8(\mathrm{CH}), 128.7(\mathrm{CH}), 129.7(2 \mathrm{CH}), 130.7(2 \mathrm{CH}), 132.5(\mathrm{CH})$, 133.0 (CH), $134.0(\mathrm{C}), 134.9(\mathrm{CH}), 136.7(\mathrm{C}), 140.1(\mathrm{C}), 142.4(\mathrm{C})$, 148.4 (C), 160.7 (C), 171.2 (C). EI-MS: $329\left(\mathrm{M}^{+}, 14\right), 238$ (12), 236 (19), 93 (78), 92 (100), 77 (52).

2.2.8. 2-((2-Nitrophenyl)amino)-6-phenoxyquinazolin-4(1H)one (8h)

Cream powder; yield: $0.31 \mathrm{~g}(82 \%)$; $\mathrm{mp}: 258-260^{\circ} \mathrm{C}$. IR $(\mathrm{KBr})\left(\nu_{\max }, \mathrm{cm}^{-1}\right): 3566,3306,1696,1665,1556,1357$, 1235, 1016. ${ }^{1} \mathrm{H}-\mathrm{NMR}\left(500 \mathrm{MHz}, \mathrm{CDCl}_{3}\right): \delta_{\mathrm{H}}=6.04(1 \mathrm{H}, \mathrm{s}$, $\mathrm{NH}), 7.26$ - 7.33 (7 H, m, $\mathrm{H}_{3,4,5}$-phenoxy, $\mathrm{H}_{4,5,6}$-phenyl, $\mathrm{H}_{5}$ quinazolinone), $7.43\left(2 \mathrm{H}, \mathrm{d},{ }^{3} \mathrm{~J}=7.8 \mathrm{~Hz}, \mathrm{H}_{2,6}\right.$-phenoxy), 7.47 (2 H, d, ${ }^{3} \mathrm{~J}=7.8 \mathrm{~Hz}, \mathrm{H}_{7,8}$-quinazolinone), 7.89 (1 H, d, ${ }^{3} \mathrm{~J}=7.5$ $\mathrm{Hz}, \mathrm{H}_{3}$-phenyl), 8.32 (1 H, s, NH). ${ }^{13} \mathrm{C}-\mathrm{NMR}(125.7 \mathrm{MHz}, \mathrm{CDCl} 3)$ : $128.9(\mathrm{CH}), 129.3(2 \mathrm{CH}), 129.7(\mathrm{CH}), 130.8(2 \mathrm{CH}), 132.8(2 \mathrm{CH})$, 133.0 (CH), 133.2 (CH), 133.5 (C), 134.1(C), 134.5 (2 CH), 138.7(C), 
140.5 (C), 142.0 (C), 148.3 (C), 160.6 (C), 170.2 (C). EI-MS: 374 $\left(\mathrm{M}^{+}, 12\right), 281$ (19), 252 (59), 137 (57), 123 (100), 93 (71).

\subsubsection{2-((4-Nitrophenyl)amino)-6-phenoxyquinazolin-4(1H)-} one (8i)

Green powder; yield: $0.33 \mathrm{~g}$ (89\%); mp: $273-275^{\circ} \mathrm{C}$. IR $(\mathrm{KBr})\left(\nu_{\max }, \mathrm{cm}^{-1}\right): 3425,3325,1688,1654,1553,1324,1236$, 1000. ${ }^{1} \mathrm{H}-\mathrm{NMR}\left(500 \mathrm{MHz}, \mathrm{CDCl}_{3}\right): \delta_{\mathrm{H}}=6.17(1 \mathrm{H}, \mathrm{s}, \mathrm{NH}), 7.29-$ 7.35 ( $5 \mathrm{H}, \mathrm{m}, \mathrm{H}_{2,4,6}$-phenoxy, $\mathrm{H}_{5,7}$-quinazolinone), $7.48(2 \mathrm{H}, \mathrm{d}$, ${ }^{3} \mathrm{~J}=7.8 \mathrm{~Hz}, \mathrm{H}_{2,6}$-phenyl), 7.82 - 7.85 (4 H, m, $\mathrm{H}_{3,5}$-phenyl, $\mathrm{H}_{3,5^{-}}$ phenoxy), $7.91\left(1 \mathrm{H}, \mathrm{d},{ }^{3} \mathrm{~J}=7.9 \mathrm{~Hz}, \mathrm{H}_{8}\right.$-quinazolinone), 9.10 (1 H, s, NH). ${ }^{13} \mathrm{C}-\mathrm{NMR}$ (125.7MHz, CDCl3): $127.4(2 \mathrm{CH}), 128.7$ (2 CH), $129.2(2 \mathrm{CH}), 130.1(\mathrm{CH}), 132.6(\mathrm{CH}), 135.7(2 \mathrm{CH}), 136.2$ (CH), 136.9 (C), $137.1(\mathrm{CH}), 138.0(\mathrm{C}), 138.7(\mathrm{C}), 140.3(\mathrm{C}), 144.7$ (C), $149.4(\mathrm{C}), 162.7(\mathrm{C}), 171.6(\mathrm{C})$. EI-MS: $374\left(\mathrm{M}^{+}, 15\right), 281(25)$, 252 (52), 137 (54), 123 (100), 93 (66).

2.2.10. 2-((3-Nitrophenyl)amino)-6-phenoxyquinazolin-4(1H)one $(8 j)$

Pale yellow powder; yield: $0.32 \mathrm{~g}$ (86\%); $\mathrm{mp}: 263-265^{\circ} \mathrm{C}$. IR $(\mathrm{KBr})\left(\nu_{\max }, \mathrm{cm}^{-1}\right): 3583,3254,1688,1627,1568,1377,1220$, 1033. ${ }^{1} \mathrm{H}-\mathrm{NMR}\left(500 \mathrm{MHz}, \mathrm{CDCl}_{3}\right): \delta_{\mathrm{H}}=6.20(1 \mathrm{H}, \mathrm{s}, \mathrm{NH}), 7.29$ 7.34 (4 H, m, $\mathrm{H}_{2,4,6}$-phenoxy, $\mathrm{H}_{7}$-quinazolinone), 7.47 (1 H, d, ${ }^{3} \mathrm{~J}=7.8 \mathrm{~Hz}, \mathrm{H}_{6}$-phenyl), 7.60 - 7.63 (5 H, m, $\mathrm{H}_{4}$-phenyl, $\mathrm{H}_{3,5^{-}}$ phenoxy, $\mathrm{H}_{5,8}$-quinazolinone), $7.74\left(1 \mathrm{H}, \mathrm{t},{ }^{3} \mathrm{~J}=7.9 \mathrm{~Hz}, \mathrm{H}_{5}-\right.$ phenyl), 8.04 (1 H, s, $\mathrm{H}_{2}$-phenyl), $9.12(1 \mathrm{H}, \mathrm{s}, \mathrm{NH}) .{ }^{13} \mathrm{C}-\mathrm{NMR}$ $\left(125.7 \mathrm{MHz}, \mathrm{CDCl}_{3}\right)$ : $127.6(\mathrm{CH}), 128.8(\mathrm{CH}), 129.3(\mathrm{CH}), 130.2(2$ $\mathrm{CH}), 130.3(2 \mathrm{CH}), 132.7(\mathrm{CH}), 135.8(2 \mathrm{CH}), 136.2(\mathrm{C}), 136.9(\mathrm{CH})$, 137.1(CH), 138.2(C),139.9(C),141.1(C),145.6(C),150.0(C),161.7 (C), 170.2 (C). EI-MS:374 ( $\left.\mathrm{M}^{+}, 10\right), 281(21), 252(50), 137(65), 123$ (100), 93 (81).

2.2.11. 2-((4-Bromophenyl)amino)-6-phenoxyquinazolin-4(1H)one ( $8 k)$

Cream powder; yield: $0.33 \mathrm{~g}$ (82\%); mp: $244-246^{\circ} \mathrm{C}$. IR $(\mathrm{KBr})\left(\nu_{\max }, \mathrm{cm}^{-1}\right): 3578,3365,1658,1623,1258,1026 .{ }^{1} \mathrm{H}-$ $\operatorname{NMR}\left(500 \mathrm{MHz}, \mathrm{CDCl}_{3}\right): \delta_{\mathrm{H}}=6.10(1 \mathrm{H}, \mathrm{s}, \mathrm{NH}), 6.92(1 \mathrm{H}$, t, ${ }^{3} \mathrm{~J}=7.8 \mathrm{~Hz}, \mathrm{H}_{4}$-phenoxy), $7.03\left(2 \mathrm{H}, \mathrm{t},{ }^{3} \mathrm{~J}=7.9 \mathrm{~Hz}, \mathrm{H}_{3,5^{-}}\right.$ phenoxy), 7.14 (2 H, d, ${ }^{3} \mathrm{~J}=7.9 \mathrm{~Hz}, \mathrm{H}_{2,6}$-phenyl), $7.24(1 \mathrm{H}$, d, ${ }^{3} \mathrm{~J}=7.9 \mathrm{~Hz}, \mathrm{H}_{7}$-quinazolinone), $7.30\left(1 \mathrm{H}, \mathrm{d},{ }^{3} \mathrm{~J}=7.9 \mathrm{~Hz}\right.$, $\mathrm{H}_{8}$-quinazolinone), 7.56 - $7.60\left(5 \mathrm{H}, \mathrm{m}, \mathrm{H}_{3,5}\right.$-phenyl, $\mathrm{H}_{2,6^{-}}$ phenoxy, $\mathrm{H}_{5}$-quinazolinone), $8.65(1 \mathrm{H}, \mathrm{s}, \mathrm{NH}) .{ }^{13} \mathrm{C}-\mathrm{NMR}$ (125.7MHz, $\left.\mathrm{CDCl}_{3}\right): 126.8(2 \mathrm{CH}), 127.8(\mathrm{CH}), 129.7(\mathrm{CH}), 130.1$ $(2 \mathrm{CH}), 133.8(\mathrm{CH}), 135.2(\mathrm{CH}), 138.0(4 \mathrm{CH}), 138.2(\mathrm{C}), 138.6(\mathrm{C})$, 139.7(C), 140.3(C), 145.7(C), 155.1(C),162.3(C), 171.2(C). EI-MS: $407\left(\mathrm{M}^{+}, 20\right), 252$ (22), 238 (34), 169 (62), 155 (100), 93 (29).

2.2.12. 2-((3,4-Dichlorophenyl)amino)-6-phenoxyquinazolin$4(1 \mathrm{H})$-one ( $8 l)$

White powder; yield: $0.36 \mathrm{~g}$ (91\%); mp: $275-277^{\circ} \mathrm{C}$. IR $(\mathrm{KBr})\left(\nu_{\max }, \mathrm{cm}^{-1}\right): 3548,3389,1688,1654,1237,1110 .{ }^{1} \mathrm{H}-\mathrm{NMR}$ $\left(500 \mathrm{MHz}, \mathrm{CDCl}_{3}\right): \delta_{\mathrm{H}}=6.00(1 \mathrm{H}, \mathrm{s}, \mathrm{NH}), 7.02\left(1 \mathrm{H}, \mathrm{t},{ }^{3} \mathrm{~J}=\right.$ $7.6 \mathrm{~Hz}, \mathrm{H}_{4}$-phenoxy), 7.05 - 7.08 (4 H, m, $\mathrm{H}_{2,6}$-phenoxy, $\mathrm{H}_{6}$ phenyl, $\mathrm{H}_{7}$-quinazolinone), 7.38 - 7.43 ( $4 \mathrm{H}, \mathrm{m}, \mathrm{H}_{3,5}$-phenoxy, $\mathrm{H}_{2}$-phenyl, $\mathrm{H}_{5}$-quinazolinone), $7.76\left(1 \mathrm{H}, \mathrm{d},{ }^{3} \mathrm{~J}=7.9 \mathrm{~Hz}, \mathrm{H}_{5}\right.$ phenyl), 7.91 (1 H, d, ${ }^{3} \mathrm{~J}=7.9 \mathrm{~Hz}, \mathrm{H}_{8}$-quinazolinone), 8.65 (1 $\mathrm{H}, \mathrm{s}, \mathrm{NH}) \cdot{ }^{13} \mathrm{C}-\mathrm{NMR}\left(125.7 \mathrm{MHz}, \mathrm{CDCl}_{3}\right): 127.9(\mathrm{CH}), 128.2(\mathrm{CH})$, 129.3(2 CH), $129.7(2 \mathrm{CH}), 129.8(\mathrm{CH}), 130.1(\mathrm{C}), 130.7(\mathrm{CH}), 138.2$ (CH), 138.6(2 CH), 139.9(C), 140.1(C), 145.2(C), 147.2(C), 149.0 (C), 155.2 (C), 165.3 (C), 174.4 (C). EI-MS: $397\left(\mathrm{M}^{+}, 2\right), 304$ (24), 252 (32), 159 (60), 145 (100), 93 (24).

2.2.13. 2-((4-Fluorophenyl)amino)-6-phenoxyquinazolin-4(1H)one $(8 m)$

Cream powder; yield: $0.27 \mathrm{~g}(78 \%)$; $\mathrm{mp}: 219-221^{\circ} \mathrm{C}$. IR $(\mathrm{KBr})\left(\nu_{\max }, \mathrm{cm}^{-1}\right): 3355,3325,1687,1625,1254,1013 .{ }^{1} \mathrm{H}-$ NMR $\left(500 \mathrm{MHz}, \mathrm{CDCl}_{3}\right): \delta_{\mathrm{H}}=6.23(1 \mathrm{H}, \mathrm{s}, \mathrm{NH}), 7.29(1 \mathrm{H}, \mathrm{t}$, ${ }^{3} \mathrm{~J}=7.8 \mathrm{~Hz}, \mathrm{H}_{4}$-phenoxy), 7.30 - 7.36 (7 H, m, $\mathrm{H}_{3,5}$-phenoxy, $\mathrm{H}_{2,3,5,6}$-phenyl, $\mathrm{H}_{5}$-quinazolinone), $7.40\left(1 \mathrm{H}, \mathrm{d},{ }^{3} \mathrm{~J}=7.9 \mathrm{~Hz}\right.$, $\mathrm{H}_{7}$-quinazolinone), 7.51 ( $2 \mathrm{H}, \mathrm{d},{ }^{3} \mathrm{~J}=7.9 \mathrm{~Hz}, \mathrm{H}_{2,6}$ - phenoxy), $7.92\left(1 \mathrm{H}, \mathrm{d},{ }^{3} \mathrm{~J}=7.9 \mathrm{~Hz}, \mathrm{H}_{8}\right.$-quinazolinone), 8.65 (1 H, s, NH). ${ }^{13} \mathrm{C}-\mathrm{NMR}\left(125.7 \mathrm{MHz}, \mathrm{CDCl}_{3}\right): 126.2(2 \mathrm{CH}), 127.5(2 \mathrm{CH}), 127.7$ $(\mathrm{CH}), 128.7(2 \mathrm{CH}), 129.2(\mathrm{CH}), 130.0(\mathrm{CH}), 130.7(\mathrm{CH}), 132.6(\mathrm{C})$, 138.6 (2 CH), 138.9 (C), 139.4 (C), $142.2(\mathrm{C}), 147.2$ (C), $150.1(\mathrm{C})$, 160.3 (C), 172.2 (C). EI-MS: $347\left(\mathrm{M}^{+}, 3\right), 254$ (25), 238 (54), 110 (62), 96 (100), 93 (45).

2.2.14. 2-((4-Chlorophenyl)amino)-6-phenoxyquinazolin-4(1H)one (8n)

Cream powder; yield: $0.31 \mathrm{~g}(86 \%)$; $\mathrm{mp}: 248-250^{\circ} \mathrm{C}$. IR $(\mathrm{KBr})\left(\nu_{\mathrm{max}}, \mathrm{cm}^{-1}\right): 3524,3325,1685,1675,1245,1019 .{ }^{1} \mathrm{H}-\mathrm{NMR}$ $\left(500 \mathrm{MHz}, \mathrm{CDCl}_{3}\right): \delta_{\mathrm{H}}=6.13(1 \mathrm{H}, \mathrm{s}, \mathrm{NH}), 6.88\left(1 \mathrm{H}, \mathrm{t},{ }^{3} \mathrm{~J}=7.8 \mathrm{~Hz}\right.$, $\mathrm{H}_{4}$-phenoxy), 7.11 (2 H, d, ${ }^{3} \mathrm{~J}=7.9 \mathrm{~Hz}, \mathrm{H}_{2,6}$-phenoxy), 7.23 - 7.26 (4 H, m, $\mathrm{H}_{2,3,5,6}$-phenyl), 7.28 - 7.35 (3 H, m, $\mathrm{H}_{3,5}$-phenoxy, $\mathrm{H}_{5}$ quinazolinone), $7.56\left(2 \mathrm{H}, \mathrm{d},{ }^{3} \mathrm{~J}=7.9 \mathrm{~Hz}, \mathrm{H}_{7,8}\right.$-quinazolinone), $8.65(1 \mathrm{H}, \mathrm{s}, \mathrm{NH}) .{ }^{13} \mathrm{C}-\mathrm{NMR}\left(125.7 \mathrm{MHz}, \mathrm{CDCl}_{3}\right): 126.6(2 \mathrm{CH})$, $129.3(\mathrm{CH}), 129.7(\mathrm{CH}), 134.0(2 \mathrm{CH}), 137.4(2 \mathrm{CH}), 138.4(\mathrm{CH})$, 138.5 (C), 138.6 (CH), $139.2(2 \mathrm{CH}), 139.5(\mathrm{C}), 139.8(\mathrm{C}), 143.4(\mathrm{C})$, 147.8 (C), 151.1 (C), 160.3 (C), 173.3 (C). EI-MS: 365 (M+2, 7), 363 (13), 252 (29), 238 (41), 126 (62), 112 (100), 93 (44).

2.2.15. 2-((3-Chlorophenyl)amino)-6-phenoxyquinazolin-4(1H)one (80)

White powder; yield: $0.31 \mathrm{~g}$ (84\%); $\mathrm{mp}: 237-239^{\circ} \mathrm{C}$. IR $(\mathrm{KBr})\left(\nu_{\max }, \mathrm{cm}^{-1}\right): 3578,3325,1688,1674,1299,1124 .{ }^{1} \mathrm{H}-$ $\operatorname{NMR}\left(500 \mathrm{MHz}, \mathrm{CDCl}_{3}\right): \delta_{\mathrm{H}}=6.02(1 \mathrm{H}, \mathrm{s}, \mathrm{NH}), 6.86(1 \mathrm{H}, \mathrm{t}$, ${ }^{3} \mathrm{~J}=7.8 \mathrm{~Hz}, \mathrm{H}_{5}$-phenyl), 7.03 - 7.06 (7 H, m, $\mathrm{H}_{2,6}$-phenyl, $\mathrm{H}_{5}$ quinazolinone, $\mathrm{H}_{2,3,5,6}$-phenoxy), $7.12\left(1 \mathrm{H}, \mathrm{d},{ }^{3} \mathrm{~J}=7.9 \mathrm{~Hz}, \mathrm{H}_{4}\right.$ phenyl), 7.19 (1 H, t, ${ }^{3} \mathrm{~J}=7.9 \mathrm{~Hz}, \mathrm{H}_{4}$-phenoxy), $7.27(1 \mathrm{H}, \mathrm{d}$, ${ }^{3} \mathrm{~J}=7.9 \mathrm{~Hz}, \mathrm{H}_{7}$-quinazolinone), $7.56\left(1 \mathrm{H}, \mathrm{d},{ }^{3} \mathrm{~J}=7.9 \mathrm{~Hz}, \mathrm{H}_{8}\right.$ quinazolinone), $8.52(1 \mathrm{H}, \mathrm{s}, \mathrm{NH}) \cdot{ }^{13} \mathrm{C}-\mathrm{NMR}\left(125.7 \mathrm{MHz}, \mathrm{CDCl}_{3}\right)$ : 126.5 (2 CH), 129.7 (CH), 129.9 (2 CH), 130.1 (2 CH), 132.6 (C), 
135.2 (CH), 136.0 (CH), 137.0 (CH), $138.1(2 \mathrm{CH}), 138.3(\mathrm{C}), 138.9$ (C), 143.4 (C), $145.2(\mathrm{C}), 148.1(\mathrm{C}), 162.5(\mathrm{C}), 172.5(\mathrm{C})$. EI-MS: 365 (M+2, 9), 363 (18), 252 (35), 238 (49), 126 (65), 112 (100), 93 (77).

\subsection{General Procedure for the Synthesis of Compounds $9 a-0$}

A mixture of 2-amino-benzoic acid $(5.0 \mathrm{mmol})$ and ethyl chloroformate $(6.0 \mathrm{mmol})$ in $\mathrm{DMF}(3 \mathrm{ml})$ was refluxed at $110^{\circ} \mathrm{C}$ for $3 \mathrm{~h} . \mathrm{Et}_{3} \mathrm{~N}(2.0 \mathrm{mmol})$ was added to the reaction dropwise over $10 \mathrm{~min}$ at room temperature and refluxed at $110{ }^{\circ} \mathrm{C}$ for $3 \mathrm{~h}$. Then, the mixture was cooled, diluted with $\mathrm{CH}_{2} \mathrm{Cl}_{2}\left(2 \mathrm{~mL}\right.$ ) and aqueous $\mathrm{NH}_{4} \mathrm{Cl}$ solution ( $3 \mathrm{~mL}$ ), and stirred for $30 \mathrm{~min}$. The aqueous layer was extracted with $\mathrm{CH}_{2} \mathrm{Cl}_{2}$, dried over sodium sulfate anhydrous, and concentrated under reduced pressure. The precipitate was recrystallized from EtOH (30 mL) to afford isatoic anhydride.

To a mixture of appropriate aniline or benzylamine (3.0 $\mathrm{mmol})$ and trichloroacetonitrile $(3.5 \mathrm{mmol})$, a mixture of synthesized isatoic anhydride (3.5 mmol) and CuO (0.1 $\mathrm{mmol}$ ) in DMF (3 $\mathrm{mL}$ ) was slowly added and refluxed at $110^{\circ} \mathrm{C}$ for $4 \mathrm{~h}$. TLC (eluent: AcOEt/ hexane 1:3) was applied to determine the end of the reaction. The mixture was diluted with $\mathrm{CH}_{2} \mathrm{Cl}_{2}(2 \mathrm{~mL})$ and aqueous $\mathrm{NH}_{4} \mathrm{Cl}$ solution ( $3 \mathrm{~mL}$ ) and stirred for $30 \mathrm{~min}$. The aqueous layer was extracted with $\mathrm{CH}_{2} \mathrm{Cl}_{2}$ dried over sodium sulfate anhydrous and concentrated under reduced pressure. The precipitate was washed with diethyl ether to afford final product 9.

\subsubsection{2-(Benzylamino)quinazolin-4(1H)-one (9a)}

White powder; yield: $0.21 \mathrm{~g}(82 \%)$ mp: $158-160^{\circ} \mathrm{C}$. IR (KBr) $\left(\nu_{\max }, \mathrm{cm}^{-1}\right): 3443,3301,1691,1677,1014 .{ }^{1} \mathrm{H}-\mathrm{NMR}$ $\left(500 \mathrm{MHz}, \mathrm{CDCl}_{3}\right): \delta_{\mathrm{H}}=4.13\left(2 \mathrm{H}, \mathrm{s}, \mathrm{CH}_{2}\right), 6.08(1 \mathrm{H}, \mathrm{s}, \mathrm{NH}), 7.26$ - 7.30 ( $3 \mathrm{H}, \mathrm{m}, \mathrm{H}_{6}$-quinazolinone, $\mathrm{H}_{3,5}$-benzylamine), 7.44 (2 $\mathrm{H}, \mathrm{d},{ }^{3} \mathrm{~J}=7.4 \mathrm{~Hz}, \mathrm{H}_{5,8}$-quinazolinone $), 7.59\left(1 \mathrm{H}, \mathrm{t},{ }^{3} \mathrm{~J}=7.9 \mathrm{~Hz}\right.$, $\mathrm{H}_{7}$-quinazolinone), $7.67\left(1 \mathrm{H}, \mathrm{t},{ }^{3} \mathrm{~J}=7.9 \mathrm{~Hz}, \mathrm{H}_{4}\right.$-benzylamine), $7.87\left(2 \mathrm{H}, \mathrm{d},{ }^{3} \mathrm{~J}=7.8 \mathrm{~Hz}, \mathrm{H}_{2,6}\right.$-benzylamine), $8.06(1 \mathrm{H}, \mathrm{s}, \mathrm{NH})$. ${ }^{13} \mathrm{C}-\mathrm{NMR}\left(125.7 \mathrm{MHz}, \mathrm{CDCl}_{3}\right): 47.6\left(\mathrm{CH}_{2}\right), 123.5(2 \mathrm{CH}), 127.0$ $(\mathrm{CH}), 127.4(\mathrm{CH}), 128.8(\mathrm{CH}), 130.2(2 \mathrm{CH}), 132.5(\mathrm{CH}), 133.1(\mathrm{C})$, $135.7(\mathrm{CH}), 144.7(\mathrm{C}), 146.0(\mathrm{C}), 161.3(\mathrm{C}), 167.0$ (C). EI-MS: 251 $\left(\mathrm{M}^{+}, 12\right), 146(33), 133(29), 119(78), 106(100), 77(25)$.

\subsubsection{2-((2-Chlorobenzyl)amino)quinazolin-4(1H)-one (9b)}

Gray powder; yield: $0.24 \mathrm{~g}$ (84\%); mp: $169-171^{\circ} \mathrm{C} . \mathrm{IR}(\mathrm{KBr})$ $\left(\nu_{\max }, \mathrm{cm}^{-1}\right): 3404,3324,1688,1671,1010 .{ }^{1} \mathrm{H}-\mathrm{NMR}(500 \mathrm{MHz}$, $\left.\mathrm{CDCl}_{3}\right): \delta_{\mathrm{H}}=4.12\left(2 \mathrm{H}, \mathrm{s}, \mathrm{CH}_{2}\right), 6.21(1 \mathrm{H}, \mathrm{s}, \mathrm{NH}), 7.29-7.35(5$ $\mathrm{H}, \mathrm{m}, \mathrm{H}_{4,5,6}$-benzylamine, $\mathrm{H}_{6,7}$-quinazolinone), $7.38(1 \mathrm{H}, \mathrm{d}$, ${ }^{3} \mathrm{~J}=7.8 \mathrm{~Hz}, \mathrm{H}_{8}$-quinazolinone), $7.50\left(1 \mathrm{H}, \mathrm{d},{ }^{3} \mathrm{~J}=7.9 \mathrm{~Hz}, \mathrm{H}_{5}-\right.$ quinazolinone), $7.91\left(1 \mathrm{H}, \mathrm{d},{ }^{3} \mathrm{~J}=7.9 \mathrm{~Hz}, \mathrm{H}_{3}\right.$-benzylamine), $8.77(1 \mathrm{H}, \mathrm{s}, \mathrm{NH}) \cdot{ }^{13} \mathrm{C}-\mathrm{NMR}\left(125.7 \mathrm{MHz}, \mathrm{CDCl}_{3}\right): 47.2\left(\mathrm{CH}_{2}\right), 125.6$ (3 CH), $127.5(2 \mathrm{CH}), 128.8(\mathrm{CH}), 129.2(\mathrm{CH}), 130.7(\mathrm{CH}), 132.5$ (C), 142.1 (C), 144.5 (C), 147.3 (C), 161.2 (C), 169.7 (C). EI-MS: 287
(M+2, 4), 285 (8), 174 (19), 167 (21), 146 (22), 126 (62), 119 (75), $111(100)$.

\subsubsection{2-((4-Methoxybenzyl)amino)quinazolin-4(1H)-one (9c)}

Cream powder; yield: $0.22 \mathrm{~g}$ (80\%); mp: $149-151^{\circ} \mathrm{C}$. IR (KBr) $\left(\nu_{\max }, \mathrm{cm}^{-1}\right): 3368,3299,1690,1663,1011 .{ }^{1} \mathrm{H}-\mathrm{NMR}$ $\left(500 \mathrm{MHz}, \mathrm{CDCl}_{3}\right): \delta_{\mathrm{H}}=3.55\left(3 \mathrm{H}, \mathrm{s}, \mathrm{OCH}_{3}\right), 4.20\left(2 \mathrm{H}, \mathrm{s}, \mathrm{CH}_{2}\right)$, $6.12(1 \mathrm{H}, \mathrm{s}, \mathrm{NH}), 7.30$ - $7.34\left(4 \mathrm{H}, \mathrm{m}, \mathrm{H}_{3,5}\right.$-benzylamine, $\mathrm{H}_{6,7^{-}}$ quinazolinone), $7.41\left(2 \mathrm{H}, \mathrm{d},{ }^{3} \mathrm{~J}=7.8 \mathrm{~Hz}, \mathrm{H}_{2,6}\right.$-benzylamine), $7.50\left(1 \mathrm{H}, \mathrm{d},{ }^{3} \mathrm{~J}=7.9 \mathrm{~Hz}, \mathrm{H}_{8}\right.$-quinazolinone $), 7.92\left(1 \mathrm{H}, \mathrm{d},{ }^{3} \mathrm{~J}=7.8\right.$ $\mathrm{Hz}, \mathrm{H}_{5}$-quinazolinone), $9.00(1 \mathrm{H}, \mathrm{s}, \mathrm{NH}) .{ }^{13} \mathrm{C}-\mathrm{NMR}(125.7 \mathrm{MHz}$, $\left.\mathrm{CDCl}_{3}\right): 47.7\left(\mathrm{CH}_{2}\right), 58.6\left(\mathrm{OCH}_{3}\right), 126.7(2 \mathrm{CH}), 127.0(\mathrm{CH}), 127.3$ $(\mathrm{CH}), 128.7(2 \mathrm{CH}), 129.7(\mathrm{CH}), 130.7(\mathrm{CH}), 132.5(\mathrm{C}), 136.7(\mathrm{C})$, 142.4 (C), 145.3 (C), 160.7 (C), 170.0 (C). EI-MS: $281\left(\mathrm{M}^{+}, 3\right), 174$ (24), 167 (44), 146 (39), 119 (100), 107 (35).

\subsubsection{2-((4-Chlorobenzyl)amino)quinazolin-4(1H)-one (9d)}

White powder; yield: $0.25 \mathrm{~g}$ (88\%); mp: $174-176^{\circ} \mathrm{C}$. IR $(\mathrm{KBr})\left(\nu_{\max }, \mathrm{cm}^{-1}\right): 3452,3300,1688,1672,1000 .{ }^{1} \mathrm{H}-\mathrm{NMR}$ $\left(500 \mathrm{MHz}, \mathrm{CDCl}_{3}\right): \delta_{\mathrm{H}}=4.15\left(2 \mathrm{H}, \mathrm{s}, \mathrm{CH}_{2}\right), 6.00(1 \mathrm{H}, \mathrm{s}, \mathrm{NH})$, 7.28 - 7.33 ( $4 \mathrm{H}, \mathrm{m}, \mathrm{H}_{2,6}$-benzylamine, $\mathrm{H}_{6,7}$-quinazolinone), $7.44\left(1 \mathrm{H}, \mathrm{d},{ }^{3} \mathrm{~J}=7.9 \mathrm{~Hz}, \mathrm{H}_{8}\right.$-quinazolinone), $7.47\left(1 \mathrm{H}, \mathrm{d},{ }^{3} \mathrm{~J}\right.$ $=8.0 \mathrm{~Hz}, \mathrm{H}_{5}$-quinazolinone), $7.89\left(2 \mathrm{H}, \mathrm{d},{ }^{3} \mathrm{~J}=7.9 \mathrm{~Hz}, \mathrm{H}_{3,5}\right.$ benzylamine), $8.57(1 \mathrm{H}, \mathrm{s}, \mathrm{NH}) .{ }^{13} \mathrm{C}-\mathrm{NMR}\left(125.7 \mathrm{MHz}, \mathrm{CDCl}_{3}\right)$ : $46.2\left(\mathrm{CH}_{2}\right), 128.9(2 \mathrm{CH}), 129.3(\mathrm{CH}), 129.7(2 \mathrm{CH}), 130.8(2 \mathrm{CH})$, $132.8(\mathrm{CH}), 132.9$ (C), 135.7 (C), $141.9(\mathrm{C}), 143.6(\mathrm{C}), 160.6(\mathrm{C})$, 170.1 (C). EI-MS: 287 (M+2, 4), 285 (9), 174 (20), 167 (29), 146 (42), 119 (55), 111 (100).

\subsubsection{2-((4-Fluorobenzyl)amino)quinazolin-4(1H)-one (9e)}

White powder; yield: $0.23 \mathrm{~g}$ (87\%); mp: $151-153^{\circ} \mathrm{C}$. IR $(\mathrm{KBr})\left(\nu_{\max }, \mathrm{cm}^{-1}\right): 3463,3365,1688,1623,1025 .{ }^{1} \mathrm{H}-\mathrm{NMR}$ $\left(500 \mathrm{MHz}, \mathrm{CDCl}_{3}\right): \delta_{\mathrm{H}}=4.05\left(2 \mathrm{H}, \mathrm{s}, \mathrm{CH}_{2}\right), 5.89(1 \mathrm{H}, \mathrm{s}, \mathrm{NH}), 7.38$ $\left(2 \mathrm{H}, \mathrm{d},{ }^{3} \mathrm{~J}=7.9 \mathrm{~Hz}, \mathrm{H}_{3,5}\right.$-benzylamine $), 7.42\left(1 \mathrm{H}, \mathrm{d},{ }^{3} \mathrm{~J}=8.0 \mathrm{~Hz}\right.$, $\mathrm{H}_{8}$-quinazolinone), 7.52 - 7.60 ( $2 \mathrm{H}, \mathrm{m}, \mathrm{H}_{6,7}$-quinazolinone), $7.80\left(1 \mathrm{H}, \mathrm{d},{ }^{3} \mathrm{~J}=8.0 \mathrm{~Hz}, \mathrm{H}_{5}\right.$-quinazolinone $), 7.93\left(2 \mathrm{H}, \mathrm{d},{ }^{3} \mathrm{~J}=7.9\right.$ $\mathrm{Hz}, \mathrm{H}_{2,6}$-benzylamine), 8.35 (1 H, s, NH). ${ }^{13} \mathrm{C}-\mathrm{NMR}(125.7 \mathrm{MHz}$, $\left.\mathrm{CDCl}_{3}\right): 42.9\left(\mathrm{CH}_{2}\right), 127.9(\mathrm{CH}), 128.2(\mathrm{CH}), 129.5(\mathrm{CH}), 130.0$ (CH), 131.4 (2 CH), 133.3 (2 CH), $135.9(\mathrm{C}), 142.8(\mathrm{C}), 144.2(\mathrm{C})$, 148.0 (C), 164.6 (C), 174.7 (C). EI-MS: $269\left(\mathrm{M}^{+}, 9\right), 174$ (15), 151 (20), 146 (31), 124 (36), 110 (100), 95 (85).

\subsubsection{2-((4-Methylbenzyl)amino)quinazolin-4(1H)-one (9f)}

White powder; yield: $0.21 \mathrm{~g}(79 \%)$; $\mathrm{mp}: 148-150^{\circ} \mathrm{C}$. IR $(\mathrm{KBr})\left(\nu_{\max }, \mathrm{cm}^{-1}\right): 3425,3256,1658,1626,1045 .{ }^{1} \mathrm{H}-\mathrm{NMR}$ $\left(500 \mathrm{MHz}, \mathrm{CDCl}_{3}\right): \delta_{\mathrm{H}}=2.67\left(3 \mathrm{H}, \mathrm{s}, \mathrm{CH}_{3}\right), 4.46\left(2 \mathrm{H}, \mathrm{s}, \mathrm{CH}_{2}\right)$, $6.19(1 \mathrm{H}, \mathrm{s}, \mathrm{NH}), 7.21\left(1 \mathrm{H}, \mathrm{d},{ }^{3} \mathrm{~J}=7.6 \mathrm{~Hz}, \mathrm{H}_{8}\right.$-quinazolinone), $7.23\left(1 \mathrm{H}, \mathrm{t},{ }^{3} \mathrm{~J}=7.6 \mathrm{~Hz}, \mathrm{H}_{6}\right.$-quinazolinone), $7.28\left(2 \mathrm{H}, \mathrm{d},{ }^{3} \mathrm{~J}\right.$ $=7.6 \mathrm{~Hz}, \mathrm{H}_{3,5}$-benzylamine $), 7.34\left(1 \mathrm{H}, \mathrm{d},{ }^{3} \mathrm{~J}=7.6 \mathrm{~Hz}, \mathrm{H}_{5}\right.$ quinazolinone), $7.41\left(1 \mathrm{H}, \mathrm{t},{ }^{3} \mathrm{~J}=7.6 \mathrm{~Hz}, \mathrm{H}_{7}\right.$-quinazolinone), $7.92\left(2 \mathrm{H}, \mathrm{d},{ }^{3} \mathrm{~J}=7.6 \mathrm{~Hz}, \mathrm{H}_{2,6}\right.$-benzylamine), 9.06 ( $\left.1 \mathrm{H}, \mathrm{s}, \mathrm{NH}\right)$. 
${ }^{13} \mathrm{C}-\mathrm{NMR}\left(125.7 \mathrm{MHz}, \mathrm{CDCl}_{3}\right): 22.2\left(\mathrm{CH}_{3}\right), 45.7\left(\mathrm{CH}_{2}\right), 125.1(\mathrm{C})$, 126.2 $(2 \mathrm{CH}), 127.5(\mathrm{CH}), 127.7(2 \mathrm{CH}), 129.1(\mathrm{C}), 130.0(\mathrm{CH}), 130.6$ $(\mathrm{CH}), 138.8(\mathrm{CH}), 140.6(\mathrm{C}), 143.4(\mathrm{C}), 160.0(\mathrm{C}), 171.1(\mathrm{C})$. EI-MS: $265\left(\mathrm{M}^{+}, 10\right), 174$ (12), 147 (42), 120 (100), 119 (33), 91 (88).

\subsubsection{2-(Phenylamino)quinazolin-4(1H)-one (9g)}

Cream powder; yield: $0.19 \mathrm{~g}(80 \%)$; $\mathrm{mp}: 144-146^{\circ} \mathrm{C}$. IR $(\mathrm{KBr})\left(\nu_{\max }, \mathrm{cm}^{-1}\right): 3410,3321,1641,1620,1055 .{ }^{1} \mathrm{H}-\mathrm{NMR}$ $\left(500 \mathrm{MHz}, \mathrm{CDCl}_{3}\right): \delta_{\mathrm{H}}=6.12(1 \mathrm{H}, \mathrm{s}, \mathrm{NH}), 7.29-7.35(3 \mathrm{H}, \mathrm{m}$, $\mathrm{H}_{6,8}$-quinazolinone, $\mathrm{H}_{4}$-phenyl), $7.48\left(2 \mathrm{H}, \mathrm{d},{ }^{3} \mathrm{~J}=7.4 \mathrm{~Hz}, \mathrm{H}_{2,6^{-}}\right.$ phenyl), 7.82-7.85 (3 H, m, $\mathrm{H}_{3,5}$-phenyl, $\mathrm{H}_{7}$-quinazolinone,), $7.91\left(1 \mathrm{H}, \mathrm{d},{ }^{3} \mathrm{~J}=7.9 \mathrm{~Hz}, \mathrm{H}_{5}\right.$-quinazolinone), $9.22(1 \mathrm{H}, \mathrm{s}, \mathrm{NH})$. ${ }^{13} \mathrm{C}-\mathrm{NMR}\left(125.7 \mathrm{MHz}, \mathrm{CDCl}_{3}\right): 127.4(2 \mathrm{CH}), 127.9(2 \mathrm{CH}), 128.7(2$ $\mathrm{CH}), 129.2(\mathrm{CH}), 130.1(\mathrm{CH}), 132.6(\mathrm{C}), 135.7(\mathrm{CH}), 144.8(\mathrm{C}), 151.5$ (C), 163.3(C), 171.6 (C). EI-MS: $237\left(\mathrm{M}^{+}, 12\right), 160(15), 146(18), 119$ (44), 92 (100), 77 (58).

\subsubsection{2-((2-Nitrophenyl)amino)quinazolin-4(1H)-one (9h)}

Gray powder; yield: 0.24 g(84\%); mp: $181-183^{\circ} \mathrm{C}$. IR(KBr) $\left(\nu_{\max }, \mathrm{cm}^{-1}\right): 3521,3341,1681,1625,1556,1346,1019 .{ }^{1} \mathrm{H}-\mathrm{NMR}$ $\left(500 \mathrm{MHz}, \mathrm{CDCl}_{3}\right): \delta_{\mathrm{H}}=6.11(1 \mathrm{H}, \mathrm{s}, \mathrm{NH}), 7.29-7.34(3 \mathrm{H}, \mathrm{m}$, $\mathrm{H}_{6,8}$-quinazolinone, $\mathrm{H}_{4}$-phenyl), $7.47\left(1 \mathrm{H}, \mathrm{d},{ }^{3} \mathrm{~J}=7.9 \mathrm{~Hz}, \mathrm{H}_{5}-\right.$ quinazolinone), 7.60 - $7.63\left(2 \mathrm{H}, \mathrm{m}, \mathrm{H}_{5,6}\right.$-phenyl), $7.74(1 \mathrm{H}$, $\mathrm{t},{ }^{3} \mathrm{~J}=8.1 \mathrm{~Hz}, \mathrm{H}_{7}$-quinazolinone $), 8.04\left(1 \mathrm{H}, \mathrm{d},{ }^{3} \mathrm{~J}=7.9 \mathrm{~Hz}, \mathrm{H}_{3}-\right.$ phenyl), $9.20(1 \mathrm{H}, \mathrm{s}, \mathrm{NH}) .{ }^{13} \mathrm{C}-\mathrm{NMR}\left(125.7 \mathrm{MHz}, \mathrm{CDCl}_{3}\right): 127.8$ $(\mathrm{CH}), 128.8(2 \mathrm{CH}), 129.0(\mathrm{CH}), 130.1(2 \mathrm{CH}), 131.1(\mathrm{CH}), 132.3(\mathrm{C})$, 135.6 (CH), $141.1(\mathrm{C}), 144.8(\mathrm{C}), 150.1(\mathrm{C}), 161.0(\mathrm{C}), 170.2(\mathrm{C})$. EIMS: $282\left(\mathrm{M}^{+}, 5\right), 164$ (12), 160 (50), 137 (52), 123 (100), 119 (70).

\subsubsection{2-((4-Nitrophenyl)amino)quinazolin-4(1H)-one (9i)}

Brown powder; yield: $0.24 \mathrm{~g}$ (86\%); mp: $175-177^{\circ} \mathrm{C}$. IR $(\mathrm{KBr})\left(\nu_{\max }, \mathrm{cm}^{-1}\right): 3410,3389,1649,1615,1554,1348,1111 .{ }^{1} \mathrm{H}-$ $\operatorname{NMR}\left(500 \mathrm{MHz}, \mathrm{CDCl}_{3}\right): \delta_{\mathrm{H}}=6.05(1 \mathrm{H}, \mathrm{s}, \mathrm{NH}), 6.92(1 \mathrm{H}, \mathrm{t}$, ${ }^{3} \mathrm{~J}=7.9 \mathrm{~Hz}, \mathrm{H}_{6}$-quinazolinone), $7.03\left(1 \mathrm{H}, \mathrm{t},{ }^{3} \mathrm{~J}=7.9 \mathrm{~Hz}, \mathrm{H}_{7^{-}}\right.$ quinazolinone), $7.14\left(2 \mathrm{H}, \mathrm{d},{ }^{3} \mathrm{~J}=8.2 \mathrm{~Hz}, \mathrm{H}_{2,6}\right.$-phenyl), 7.22 (1 $\mathrm{H}, \mathrm{d},{ }^{3} \mathrm{~J}=7.9 \mathrm{~Hz}, \mathrm{H}_{8}$-quinazolinone $), 7.32\left(1 \mathrm{H}, \mathrm{d},{ }^{3} \mathrm{~J}=7.9 \mathrm{~Hz}, \mathrm{H}_{5}-\right.$ quinazolinone), $7.57\left(2 \mathrm{H}, \mathrm{d},{ }^{3} \mathrm{~J}=8.2 \mathrm{~Hz}, \mathrm{H}_{3,5}\right.$-phenyl), 8.63 (1 $\mathrm{H}, \mathrm{s}, \mathrm{NH}) \cdot{ }^{13} \mathrm{C}-\mathrm{NMR}\left(125.7 \mathrm{MHz}, \mathrm{CDCl}_{3}\right): 126.7(2 \mathrm{CH}), 129.8(\mathrm{CH})$, 129.9(CH), 130.1 (2 CH), 133.1(CH), 133.9(C), 138.3(C), 138.9(C), 145.3 (C), 155.0 (C), 162.1 (C), 172.3 (C). EI-MS: $282\left(\mathrm{M}^{+}, 10\right), 164$ (19), 160 (42), 137 (25), 123 (100), 119 (42).

\subsubsection{2-((3-Nitrophenyl)amino)quinazolin-4(1H)-one (9j)}

Green powder; yield: $0.23 \mathrm{~g}$ (83\%); mp: $169-171^{\circ} \mathrm{C}$. IR $(\mathrm{KBr})\left(\nu_{\max }, \mathrm{cm}^{-1}\right): 3421,3315,1687,1658,1554,1327,1113 .{ }^{1} \mathrm{H}-$ $\operatorname{NMR}\left(500 \mathrm{MHz}, \mathrm{CDCl}_{3}\right): \delta_{\mathrm{H}}=6.00(1 \mathrm{H}, \mathrm{s}, \mathrm{NH}), 7.02(1 \mathrm{H}, \mathrm{t}$, ${ }^{3} \mathrm{~J}=7.9 \mathrm{~Hz}, \mathrm{H}_{6}$-quinazolinone), $7.07\left(1 \mathrm{H}, \mathrm{t},{ }^{3} \mathrm{~J}=8.1 \mathrm{~Hz}, \mathrm{H}_{7^{-}}\right.$ quinazolinone), 7.38 - $7.43\left(4 \mathrm{H}, \mathrm{m}, \mathrm{H}_{5,8}\right.$-quinazolinone, $\mathrm{H}_{5,6^{-}}$ phenyl), $7.74\left(1 \mathrm{H}, \mathrm{d},{ }^{3} \mathrm{~J}=7.9 \mathrm{~Hz}, \mathrm{H}_{4}\right.$-phenyl), $7.91\left(1 \mathrm{H}, \mathrm{s}, \mathrm{H}_{2^{-}}\right.$ phenyl), 8.48 (1 H, s, NH). ${ }^{13} \mathrm{C}-\mathrm{NMR}\left(125.7 \mathrm{MHz}, \mathrm{CDCl}_{3}\right): 127.9$ $(\mathrm{CH}), 128.2(\mathrm{CH}), 128.3(\mathrm{CH}), 129.0(\mathrm{CH}), 129.3(\mathrm{CH}), 129.7(\mathrm{CH})$,
129.8 (C), $130.1(\mathrm{CH}), 130.7(\mathrm{CH}), 140.1(\mathrm{C}), 147.2(\mathrm{C}), 155.2(\mathrm{C})$, 167.2 (C), 174.3 (C). EI-MS: $282\left(\mathrm{M}^{+}, 12\right), 164$ (25), 160 (54), 137 (59), 123 (100), 119 (66).

\subsubsection{2-((4-Bromophenyl)amino)quinazolin-4(1H)-one ( $9 k)$}

White powder; yield: $0.27 \mathrm{~g}(85 \%)$; $\mathrm{mp}: 164-166^{\circ} \mathrm{C}$. IR (KBr) $\left(\nu_{\max }, \mathrm{cm}^{-1}\right): 3524,3245,1656,1627,1124 .{ }^{1} \mathrm{H}-\mathrm{NMR}$ $\left(500 \mathrm{MHz}, \mathrm{CDCl}_{3}\right): \delta_{\mathrm{H}}=5.85(1 \mathrm{H}, \mathrm{s}, \mathrm{NH}), 6.89\left(1 \mathrm{H}, \mathrm{t},{ }^{3} \mathrm{~J}=7.9\right.$ $\mathrm{Hz}, \mathrm{H}_{6}$-quinazolinone), $7.12\left(2 \mathrm{H}, \mathrm{d},{ }^{3} \mathrm{~J}=7.8 \mathrm{~Hz}, \mathrm{H}_{2,6}\right.$-phenyl), 7.23 - 7.35 ( $3 \mathrm{H}, \mathrm{m}, \mathrm{H}_{3,5}$-phenyl, $\mathrm{H}_{7}$-quinazolinone), $7.56(2 \mathrm{H}$, $\mathrm{d},{ }^{3} \mathrm{~J}=7.8 \mathrm{~Hz}, \mathrm{H}_{5,8}$-quinazolinone), $8.53(1 \mathrm{H}, \mathrm{s}, \mathrm{NH}) .{ }^{13} \mathrm{C}$-NMR (125.7MHz, $\left.\mathrm{CDCl}_{3}\right): 126.6(\mathrm{CH}), 127.2(\mathrm{CH}), 129.7(2 \mathrm{CH}), 129.8(2$ $\mathrm{CH}), 130.0(\mathrm{CH}), 131.1(\mathrm{C}), 133.0(\mathrm{CH}), 138.3(\mathrm{C}), 139.0(\mathrm{C}), 145.2$ (C), 160.0 (C), 172.0 (C). EI-MS: $315\left(\mathrm{M}^{+}, 3\right), 196$ (55), 160 (33), 155 (69), 119 (100).

2.3.12. 2-((3,4-Dichlorophenyl)amino)quinazolin-4(1H)-one (9l)

White powder; yield: $0.27 \mathrm{~g}$ (90\%); mp: $191-193^{\circ} \mathrm{C}$. IR $(\mathrm{KBr})\left(\nu_{\max }, \mathrm{cm}^{-1}\right): 3356,3309,1685,1672,1015 .{ }^{1} \mathrm{H}-\mathrm{NMR}$ $\left(500 \mathrm{MHz}, \mathrm{CDCl}_{3}\right): \delta_{\mathrm{H}}=6.03(1 \mathrm{H}, \mathrm{s}, \mathrm{NH}), 6.87\left(1 \mathrm{H}, \mathrm{t},{ }^{3} \mathrm{~J}=7.9\right.$ $\mathrm{Hz}, \mathrm{H}_{6}$-quinazolinone), 7.03 (1 H, s, $\mathrm{H}_{2}$-phenyl), 7.11 (1 H, d, ${ }^{3} \mathrm{~J}=7.9 \mathrm{~Hz}, \mathrm{H}_{8}$-quinazolinone), $7.18\left(1 \mathrm{H}, \mathrm{t},{ }^{3} \mathrm{~J}=7.9 \mathrm{~Hz}, \mathrm{H}_{7^{-}}\right.$ quinazolinone), $7.27\left(1 \mathrm{H}, \mathrm{d},{ }^{3} \mathrm{~J}=7.9 \mathrm{~Hz}, \mathrm{H}_{6}\right.$-phenyl), 7.29 (1 $\mathrm{H}, \mathrm{d},{ }^{3} \mathrm{~J}=8.3 \mathrm{~Hz}, \mathrm{H}_{5}$-phenyl), $7.55\left(1 \mathrm{H}, \mathrm{d},{ }^{3} \mathrm{~J}=8.3 \mathrm{~Hz}, \mathrm{H}_{5}-\right.$ quinazolinone), $8.52(1 \mathrm{H}, \mathrm{s}, \mathrm{NH}) \cdot{ }^{13} \mathrm{C}-\mathrm{NMR}\left(125.7 \mathrm{MHz}, \mathrm{CDCl}_{3}\right)$ : 126.7 (CH), 129.7 (2 CH), 129.8 (2 CH), 130.1(C), $133.0(\mathrm{CH}), 138.3$ (2 CH), 139.0 (C), 140.0 (C),145.3(C),162.9(C),172.9(C). EI-MS: $305\left(\mathrm{M}^{+}, 1\right), 186(25), 160(23), 159$ (64), 146 (100), 119 (32).

\subsubsection{2-((4-Fluorophenyl)amino)quinazolin-4(1H)-one ( $9 \mathrm{~m})$}

Cream powder; yield: $0.20 \mathrm{~g}$ (79\%); mp: $143-145^{\circ} \mathrm{C}$. IR $(\mathrm{KBr})\left(\nu_{\max }, \mathrm{cm}^{-1}\right): 3547,3399,1651,1627,1019 .{ }^{1} \mathrm{H}-\mathrm{NMR}$ $\left(500 \mathrm{MHz}, \mathrm{CDCl}_{3}\right): \delta_{\mathrm{H}}=6.05(1 \mathrm{H}, \mathrm{s}, \mathrm{NH}), 7.28\left(1 \mathrm{H}, \mathrm{t},{ }^{3} \mathrm{~J}=7.9\right.$ $\mathrm{Hz}, \mathrm{H}_{6}$-quinazolinone), 7.30 - 7.35 (3 H, m, $\mathrm{H}_{3,5}$-phenyl, $\mathrm{H}_{7}$ quinazolinone), $7.36\left(1 \mathrm{H}, \mathrm{d},{ }^{3} \mathrm{~J}=7.9 \mathrm{~Hz}, \mathrm{H}_{8}\right.$-quinazolinone), $7.50\left(2 \mathrm{H}, \mathrm{d},{ }^{3} \mathrm{~J}=7.8 \mathrm{~Hz}, \mathrm{H}_{2,6}\right.$-phenyl), $7.92\left(1 \mathrm{H}, \mathrm{d},{ }^{3} \mathrm{~J}=7.9\right.$ $\mathrm{Hz}, \mathrm{H}_{5}$-quinazolinone), $8.53(1 \mathrm{H}, \mathrm{s}, \mathrm{NH}) .{ }^{13} \mathrm{C}-\mathrm{NMR}(125.7 \mathrm{MHz}$, $\left.\mathrm{CDCl}_{3}\right)$ : $126.2(2 \mathrm{CH}), 127.5(\mathrm{CH}), 127.7(2 \mathrm{CH}), 128.7(\mathrm{CH}), 129.2$ (CH), 130.7 (C), $132.6(\mathrm{CH}), 142.2(\mathrm{C}), 147.2(\mathrm{C}), 152.2(\mathrm{C}), 160.2$ (C), 172.2 (C). EI-MS: $255\left(\mathrm{M}^{+}, 11\right), 160$ (20), $146(21), 137(62), 110$ (100), 96 (25).

\subsubsection{2-((4-Chlorophenyl)amino)quinazolin-4(1H)-one (9n)}

White powder; yield: $0.23 \mathrm{~g}(84 \%)$ mp: $160-162^{\circ} \mathrm{C}$. IR (KBr) $\left(\nu_{\max }, \mathrm{cm}^{-1}\right): 3425,3308,1671,1625,1025 .{ }^{1} \mathrm{H}-\mathrm{NMR}$ $\left(500 \mathrm{MHz}, \mathrm{CDCl}_{3}\right): \delta_{\mathrm{H}}=5.85(1 \mathrm{H}, \mathrm{s}, \mathrm{NH}), 6.92\left(1 \mathrm{H}, \mathrm{t},{ }^{3} \mathrm{~J}\right.$ = $7.9 \mathrm{~Hz}, \mathrm{H}_{6}$-quinazolinone), $7.17\left(1 \mathrm{H}, \mathrm{d},{ }^{3} \mathrm{~J}=7.9 \mathrm{~Hz}, \mathrm{H}_{8}\right.$ quinazolinone), $7.23\left(1 \mathrm{H}, \mathrm{d},{ }^{3} \mathrm{~J}=7.8 \mathrm{~Hz}, \mathrm{H}_{5}\right.$-quinazolinone), $7.35\left(1 \mathrm{H}, \mathrm{t},{ }^{3} \mathrm{~J}=7.9 \mathrm{~Hz}, \mathrm{H}_{7}\right.$-quinazolinone), 7.56 - $7.60(4 \mathrm{H}, \mathrm{m}$, $\mathrm{H}_{2,3,5,6}$-phenyl), 8.53 (1 H, s, NH). ${ }^{13} \mathrm{C}-\mathrm{NMR}\left(125.7 \mathrm{MHz}, \mathrm{CDCl}_{3}\right.$ ): $126.8(2 \mathrm{CH}), 129.3(2 \mathrm{CH}), 129.8(\mathrm{CH}), 130.2(\mathrm{CH}), 130.4(\mathrm{CH})$, 
132.6 (C), 133.2 (CH), 142.2 (C), 145.4 (C), 148.0 (C), 160.0 (C), 170.2 (C). EI-MS: 273 (M+2, 5), 271 (11), 153 (25), 146 (24), 126 (60), 119 (100), 112 (44).

\subsubsection{2-((3-Chlorophenyl)amino)quinazolin-4(1H)-one (90)}

White powder; yield: $0.21 \mathrm{~g}(78 \%)$; $\mathrm{mp}: 153-155^{\circ} \mathrm{C}$. IR $(\mathrm{KBr})\left(\nu_{\max }, \mathrm{cm}^{-1}\right): 3448,3325,1685,1627,1015 .{ }^{1} \mathrm{H}-\mathrm{NMR}$ $\left(500 \mathrm{MHz}, \mathrm{CDCl}_{3}\right): \delta_{\mathrm{H}}=6.05(1 \mathrm{H}, \mathrm{s}, \mathrm{NH}), 7.26\left(1 \mathrm{H}, \mathrm{t},{ }^{3} \mathrm{~J}=7.9\right.$ $\mathrm{Hz}, \mathrm{H}_{6}$-quinazolinone), 7.28 - 7.35 (3 $\mathrm{H}, \mathrm{m}, \mathrm{H}_{2,4,6}$-phenyl), 7.51 $\left(1 \mathrm{H}, \mathrm{d},{ }^{3} \mathrm{~J}=7.9 \mathrm{~Hz}, \mathrm{H}_{8}\right.$-quinazolinone), $7.62\left(1 \mathrm{H}, \mathrm{t},{ }^{3} \mathrm{~J}=7.9 \mathrm{~Hz}\right.$, $\mathrm{H}_{5}$-phenyl), $7.74\left(1 \mathrm{H}, \mathrm{t},{ }^{3} \mathrm{~J}=7.9 \mathrm{~Hz}, \mathrm{H}_{7}\right.$-quinazolinone), 8.05 $\left(1 \mathrm{H}, \mathrm{d},{ }^{3} \mathrm{~J}=7.9 \mathrm{~Hz}, \mathrm{H}_{5}\right.$-quinazolinone), $8.88(1 \mathrm{H}, \mathrm{s}, \mathrm{NH}) .{ }^{13} \mathrm{C}$ $\operatorname{NMR}\left(125.7 \mathrm{MHz}, \mathrm{CDCl}_{3}\right)$ : $126.2(2 \mathrm{CH}), 127.8(\mathrm{CH}), 128.7(\mathrm{CH})$, 129.2 (CH), $130.0(\mathrm{CH}), 130.1$ (2 CH), 132.5 (C), 135.7 (C), 144.9 (C), $155.1(\mathrm{C}), 166.2$ (C), 174.9 (C). EI-MS: 273 (M+2, 7), 271 (15), $153(28), 146$ (20), 126 (68), 119 (100), 112 (40).

\subsection{Biological Evaluations}

The biological activities of the synthesized compounds, 8a-o and 9a-o, were carried out using EGFR-TK assay kit (BPS biosciences) and kinase-Glo luminescent assay (Promega). In order to start the enzymatic reaction, $\mathrm{EGFR}^{\mathrm{T} 790 \mathrm{M}}$ and their substrates were incubated with the synthesized compounds in kinase assay buffer for $60 \mathrm{~min}$ at room temperature. Then detection reagent (ADP-Glo ${ }^{\mathrm{TM}}$ reagent) was added to each well, followed by incubation at room temperature for $40 \mathrm{~min}$. With the addition of kinase detection reagent, the mixture was incubated for further $30 \mathrm{~min}$. The $\mathrm{IC}_{50}$ values were determined after the luminescence record, and all samples and controls were tested in triplicate.

\subsection{Docking Study}

Crystallographic structure of EGFR tyrosine kinase was retrieved from Protein Data Bank (PDB code: 4G5P, resolution $3.17 \AA$ ). The docking analysis was performed using AutoDock Tools version 1.5.6rc3 (http://mgltools.scripps.edu) software to evaluate the binding mode of the compound $8 \mathrm{~b}$ against $\mathrm{EGFR}^{\mathrm{T} 790 \mathrm{M}}$. At first, the original ligand and water molecules were removed, and polar hydrogen atoms and Kollman united partial atom charges were added onto the protein. The 2D structure of the synthesized compound and erlotinib were sketched using ChemBioDraw Ultra 12.0 and optimized by $\mathrm{MM}+$ force field using HyperChem8 (http://www.hyper.com). Each docking system was performed by 100 runs of the Autodock using the Lamarckian genetic algorithm (LGA). The lowest dockingenergy conformation of the highest populated cluster was considered the most stable orientation and selected for analysis. Finally, a cluster analysis was performed on the docking results using a root mean square deviation (RMSD) of $0.5 \AA$. Graphic manipulations and visualizations were done by Pymol software version 1.5.0.1 (http://pymol.findmysoft.com).

\subsection{ADME Properties}

The ADME properties of the synthesized compounds were predicted using the Molinspiration online property calculation toolkit (Molinspiration, 2018). Topological polar surface area (TPSA), number of rotatable bonds (nROTB), molecular volume (MV), molecular weight (MW), the logarithm of the partition coefficient (miLog P), number of hydrogen bond acceptors (n-ON), number of hydrogen bond donors (n-OHNH), and Lipinski's rule of five were calculated (23). Additionally, intestinal absorption (\% ABS) was calculated according to the following equation: $\mathrm{ABS} \%$ $=109-(0.345 \times$ TPSA $)(24)$.

\section{Results and Discussion}

\subsection{Chemistry}

Figure 3 displayed the general synthetic pathway of the novel compounds. 2-Amino-5-fluorobenzoic acid 1 was reacted with phenol 2 through a nucleophilic aromatic substitution using sodium hydride as the base under reflux conditions to produce compound 3. Isatoic anhydride derivatives $(5,6)$ were achieved by reacting the compounds 3 and 2-aminobenzoic acid 4 with ethyl chloroformate and triethylamine. The final products $8(\mathrm{a}-\mathrm{o})$ and $9(\mathrm{a}-\mathrm{o})$ were afforded by the treatment of the above isatoic anhydrides with corresponding aniline or benzylamine in the presence of trichloroacetonitrile and catalytic amount of $\mathrm{CuO}$ under reflux condition (25).

\subsection{Biological Evaluations}

The inhibitory activities of the novel compounds were assessed using EGFR $^{\mathrm{T} 790 \mathrm{M}} / \mathrm{ADP}^{-G l o}{ }^{\mathrm{TM}}$ kinase assay system. Erlotinib, a reversible EGFR-TKI, was used as a standard compound. As shown in Figure 4, most synthesized compounds provided nanomolar range inhibition against EGFR-TK. Some derivatives of compound 8 (i.e., compounds $8 \mathrm{a}, 8 \mathrm{~b}, 8 \mathrm{c}, 8 \mathrm{~d}$ and $8 \mathrm{f}$ ) exhibited higher inhibitory activities than the other compounds, compared to erlotinib $\left(\mathrm{IC}_{50}=\right.$ $1.5 \mathrm{nM})$. The most potent compound was $8 \mathrm{~b}$, with an $\mathrm{IC}_{50}$ value of $1.37 \mathrm{nM}$. By comparing the $\mathrm{IC}_{50}$ values of compounds $8 \mathrm{~b}$ and $9 \mathrm{~b}$, it can be concluded that introducing a phenoxy group at the 6-position could be beneficial for the activity owing to the proper fitting of the compound into a hydrophobic pocket. According to compounds 8c, 8f, and 


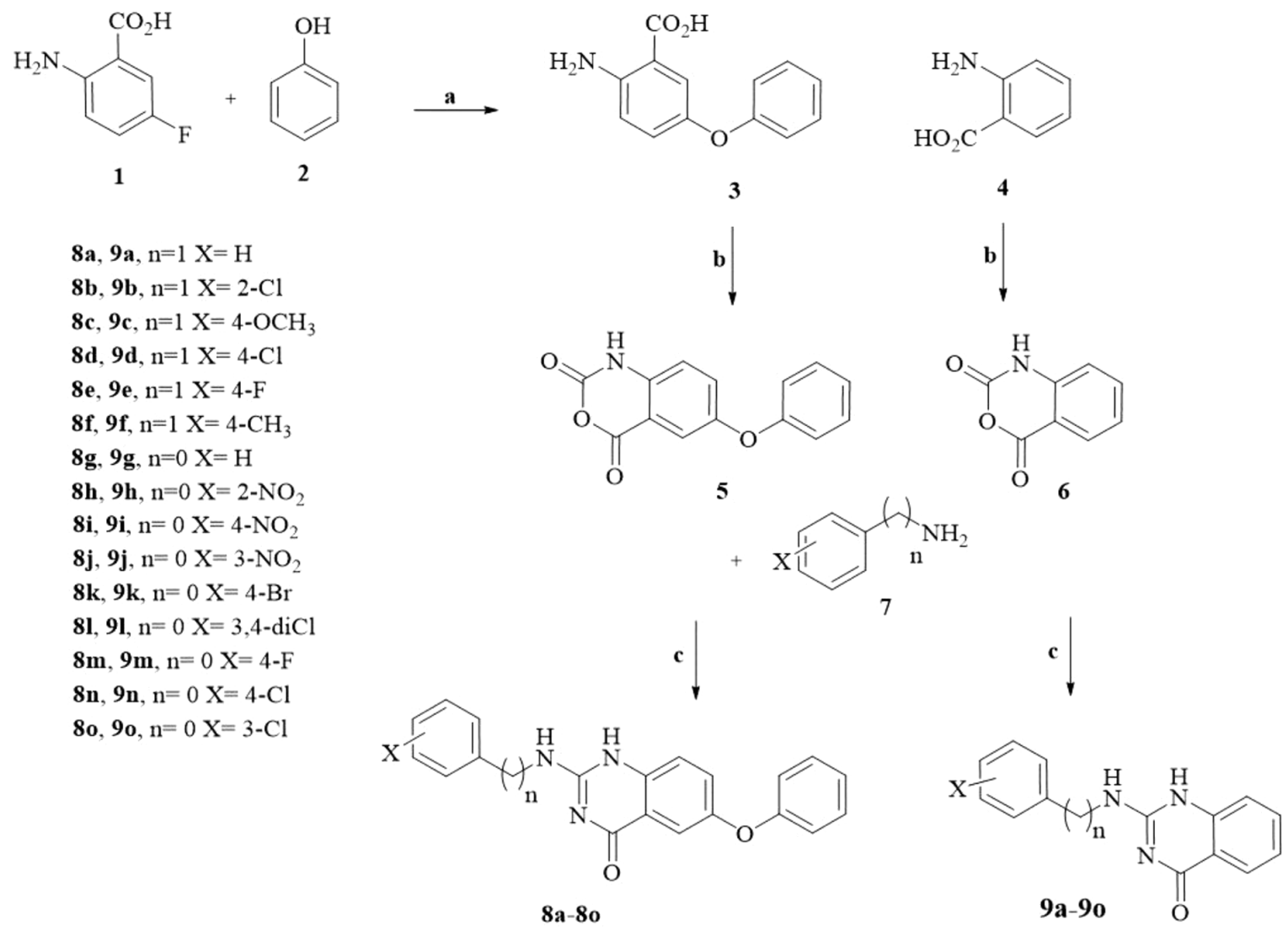

Figure 3. Reagents and condition: (A) NaH, CuI (10 mol\%), DMF, reflux, $2 \mathrm{~h}$; (B) Ethyl chloroformate, $\mathrm{Et}{ }_{3} \mathrm{~N}, \mathrm{DM}$, reflux, 3h; (C) $\mathrm{CCl}_{3} \mathrm{CN}, \mathrm{CuO}$ (10 mol\%), DMF, reflux, $4 \mathrm{~h}$.

9c, the presence of the electron-donating substituents in para position of benzyl ring in phenoxy series were more desirable than electron-withdrawing substituents; however, this finding cannot be extended to ortho position as compound $8 \mathrm{~b}$ exhibited the highest activity with an electron withdrawing substituent. Substitution of fluorine in para position of either benzyl or phenyl ring (i.e., compounds $8 \mathrm{~m}$ and $9 \mathrm{~m}$ ) was not favorable for the activity since fluorine, a hydrophilic atom could be surrounded by water molecules, leading to a difficult location into the hydrophobic pocket of the enzyme.

Moreover, compounds 8j and 9j possessing 3-nitro substituent on phenyl ring were potent analogs in both series. It could be explained that the nitro group could afford a hydrogen bond with polar amino acids in the active site. Generally, due to close variations of $\mathrm{IC}_{50} \mathrm{~s}$, the judgment about the activity trend was a challenge we were encountered, and we did not examine electron-donating substituents in phenyl bearing series, which is a limitation for our study.

\subsection{Docking Study}

A docking study was performed to identify the binding mode of compound $8 \mathrm{~b}$ and EGFR-TK active site. As shown in Figure 5, docking study revealed that this structure had a good affinity to the active site of the enzyme and fitted properly. The amide group of quinazolinone moiety and nitrogen in the hydrophobic head had suitable distances from Met793 for hydrogen bonding interaction. Additional hydrogen bonds formed between the oxygen of the phenoxy group and residues of Met790 and Thr854. As compound 8b was surrounded by lipophilic amino acids, hydrophobic interactions were observed between $8 \mathrm{~b}$ and Lys745, Val726, Leu718, Ile744. Furthermore, the superimposition of compound $8 \mathrm{~b}$ and erlotinib demonstrated that these two structures were located in the same direction in the active site as the nitrogen atoms at the 1-position of erlotinib and 3-position of $8 \mathrm{~b}$ were completely aligned and formed hydrogen bonds with Met793. 


\begin{tabular}{|c|c|c|c|c|c|c|c|}
\hline & & & & & & (a- & \\
\hline Number & $\mathbf{X}$ & n & $\mathrm{IC}_{50(\mathrm{nM})}$ & Number & $\mathbf{X}$ & n & $\mathrm{IC}_{50(\mathrm{nM})}$ \\
\hline $8 a$ & $\mathrm{H}$ & 1 & 1.93 & $9 a$ & $\mathrm{H}$ & 1 & 6.70 \\
\hline $8 \mathrm{~b}$ & $2-\mathrm{Cl}$ & 1 & 1.37 & $9 \mathrm{~b}$ & $2-\mathrm{Cl}$ & 1 & 10.24 \\
\hline $8 c$ & $4-\mathrm{OCH}_{3}$ & 1 & 1.84 & $9 c$ & $4-\mathrm{OCH}_{3}$ & 1 & 3.90 \\
\hline $8 \mathrm{~d}$ & $4-\mathrm{Cl}$ & 1 & 1.96 & $9 d$ & $4-\mathrm{Cl}$ & 1 & 5.23 \\
\hline $8 \mathrm{e}$ & $4-\mathrm{F}$ & 1 & 5.00 & $9 \mathrm{e}$ & $4-F$ & 1 & 6.36 \\
\hline $8 \mathrm{f}$ & $4-\mathrm{CH}_{3}$ & 1 & 1.55 & $9 f$ & $4-\mathrm{CH}_{3}$ & 1 & 5.49 \\
\hline $8 g$ & $\mathrm{H}$ & 0 & $\mathrm{NA}^{\mathrm{a}}$ & $9 \mathrm{~g}$ & $\mathrm{H}$ & 0 & 6.94 \\
\hline $8 \mathrm{~h}$ & $2-\mathrm{NO}_{2}$ & 0 & 13.80 & $9 \mathrm{~h}$ & $2-\mathrm{NO}_{2}$ & 0 & 6.07 \\
\hline $8 \mathrm{i}$ & $4-\mathrm{NO}_{2}$ & 0 & 15.69 & $9 \mathrm{i}$ & $4-\mathrm{NO}_{2}$ & 0 & 4.17 \\
\hline $8 \mathrm{j}$ & $3-\mathrm{NO}_{2}$ & 0 & 13.50 & $9 \mathrm{j}$ & $3-\mathrm{NO}_{2}$ & 0 & 2.80 \\
\hline $8 \mathrm{k}$ & $4-\mathrm{Br}$ & 0 & 15.64 & $9 \mathrm{k}$ & $4-\mathrm{Br}$ & 0 & 5.95 \\
\hline 81 & 3,4-diCl & 0 & 14.03 & 91 & 3,4-diCl & 0 & 6.49 \\
\hline $8 \mathrm{~m}$ & $4-\mathrm{F}$ & 0 & $\mathrm{NA}^{\mathrm{a}}$ & $9 m$ & $4-F$ & 0 & 57.40 \\
\hline $8 \mathrm{n}$ & $4-\mathrm{Cl}$ & 0 & 14.50 & $9 n$ & $4-\mathrm{Cl}$ & 0 & 8.74 \\
\hline 80 & $3-\mathrm{Cl}$ & 0 & 14.02 & 90 & $3-\mathrm{Cl}$ & 0 & 6.40 \\
\hline Erlotinib & - & - & 1.50 & & & & \\
\hline
\end{tabular}

Figure 4. Inhibitory activities of novel quinazolin-4(1H)-one derivatives against EGFR tyrosine kinase (NA: no activity).

\subsection{ADME Properties}

To predict oral bioavailability and druglikeness of our novel compounds, ADME calculations were performed using molinspiration calculator (Table 1). According to the Lipinski's rule of five (miLog $\mathrm{P}$ (octanol-water partition coefficient $) \leq 5$, molecular weight $\leq 500$, number of hydrogen bond acceptors $\leq 10$, and number of hydrogen bond donors $\leq 5$ ), an orally active drug candidate has no more than one violation of the criteria (26). All the synthesized compounds exhibited an excellent ABS\% ranging from $70.07 \%$ to $89.06 \%$. They could be orally active agents through fulfilling the criteria.

\subsection{Conclusions}

In summary, we designed and synthesized thirty qinazolinone derivatives as EGFR-TKIs. All the compounds were evaluated for their inhibitory activities against EGFR ${ }^{\mathrm{T} 790 \mathrm{M}}$. Most of our novel compounds provided nanomolar range inhibition. Compounds 8a, 8b, 8c, 8d, and 8f exhibited more potent inhibitory activities than erlotinib. Remarkably, Compound 8b with 2-chlorobenzyl group in phenoxy derivatives showed the exemplary inhibitory activity with an $\mathrm{IC}_{50}$ value of $0.37 \mathrm{nM}$. The docking study of $8 \mathrm{~b}$ demonstrated that it had a good affinity to the active site of the enzyme and fitted properly. According to our findings, at- 


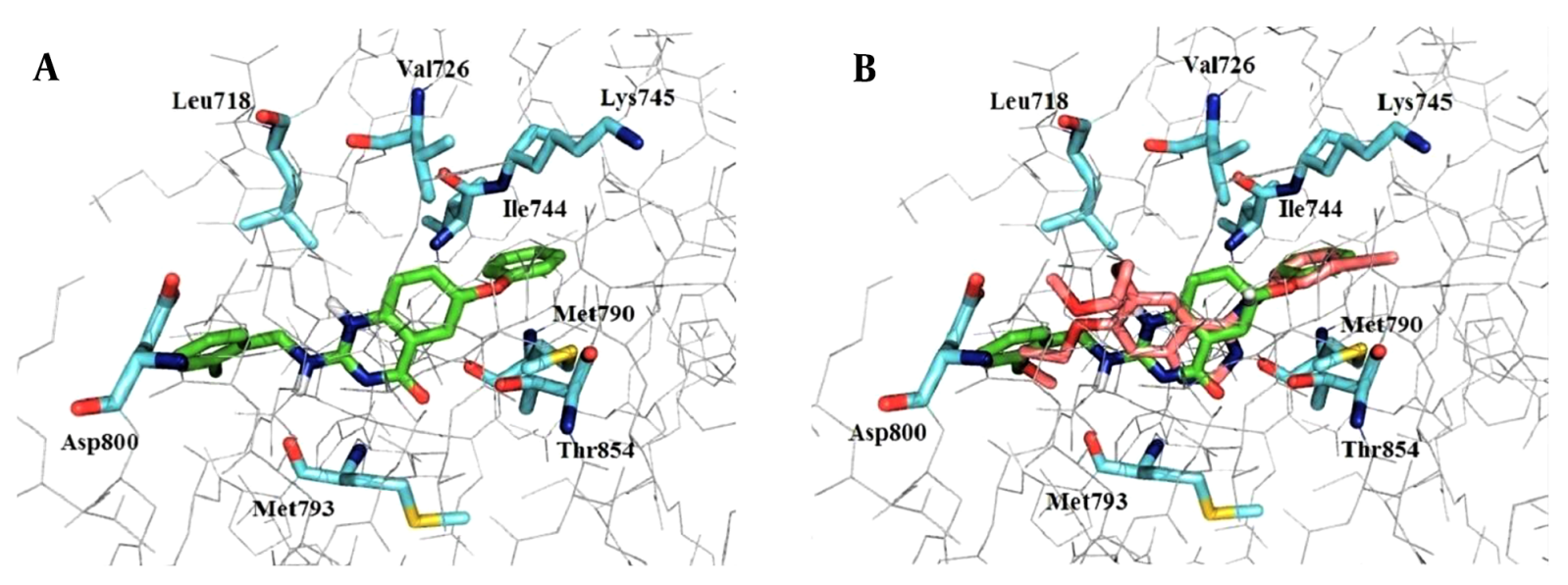

Figure 5. (A) Compound $8 \mathrm{~b}$ (green sticks) in the active site of $\mathrm{EGFR}^{\mathrm{T} 790 \mathrm{M}}$ receptor (amino acid residues in blue PDB: 4G5P). The amide group of quinazolinone moiety and nitrogen in the hydrophobic head had suitable distances from Met793 for hydrogen bonding interaction. Additional hydrogen bond formed between oxygen of phenoxy group and Met790 and Thr854. The hydrophobic interactions observed between 8b and Lys745, Val726, Leu718, and Ile744. (B) The superimposition of compound $8 \mathrm{~b}$ (green sticks) and erlotinib (salmon pink sticks) in the active site of $\mathrm{EGFR}^{\mathrm{T} 790 \mathrm{M}}$ receptor.

\begin{tabular}{|c|c|c|c|c|c|c|c|c|c|}
\hline Number & $\%$ ABS & $\operatorname{TPSA}\left(\mathbf{A}^{2}\right)$ & n ROTB & MV & мw & milogP & n-ON Acceptors & n-OHNH Donors & Lipinski's Violations \\
\hline $\mathbf{8 a}$ & 85.88 & 67.02 & 5 & 308.85 & 343.39 & 4.71 & 5 & 2 & 0 \\
\hline $8 b$ & 85.88 & 67.02 & 5 & 322.39 & 377.83 & 5.34 & 5 & 2 & 1 \\
\hline $8 c$ & 82.69 & 76.25 & 6 & 334.40 & 373.41 & 4.77 & 6 & 2 & 0 \\
\hline $8 d$ & 85.88 & 67.02 & 5 & 322.39 & 377.83 & 5.39 & 5 & 2 & 1 \\
\hline $8 \mathrm{e}$ & 85.88 & 67.02 & 5 & 313.79 & 361.38 & 4.88 & 5 & 2 & 0 \\
\hline $8 f$ & 85.88 & 67.02 & 5 & 325.42 & 357.41 & 5.16 & 5 & 2 & 1 \\
\hline $8 g$ & 85.88 & 67.02 & 4 & 292.05 & 329.36 & 5.03 & 5 & 2 & 1 \\
\hline $8 \mathrm{~h}$ & 70.07 & 112.84 & 5 & 315.39 & 374.36 & 4.94 & 8 & 2 & 0 \\
\hline $8 \mathrm{i}$ & 70.07 & 112.84 & 5 & 315.39 & 374.36 & 4.99 & 8 & 2 & 0 \\
\hline $8 \mathbf{j}$ & 70.07 & 112.84 & 5 & 315.39 & 374.36 & 4.96 & 8 & 2 & 0 \\
\hline $8 k$ & 85.88 & 67.02 & 4 & 309.94 & 408.25 & 5.84 & 5 & 2 & 1 \\
\hline 81 & 85.88 & 67.02 & 4 & 319.12 & 398.25 & 6.31 & 5 & 2 & 1 \\
\hline $8 m$ & 85.88 & 67.02 & 4 & 296.98 & 347.35 & 5.19 & 5 & 2 & 1 \\
\hline $8 n$ & 85.88 & 67.02 & 4 & 305.59 & 363.80 & 5.71 & 5 & 2 & 1 \\
\hline 80 & 85.88 & 67.02 & 4 & 305.59 & 363.80 & 5.68 & 5 & 2 & 1 \\
\hline $9 a$ & 89.06 & 57.78 & 3 & 228.46 & 251.29 & 2.98 & 4 & 2 & 0 \\
\hline 9b & 89.06 & 57.78 & 3 & 242.00 & 285.73 & 3.61 & 4 & 2 & 0 \\
\hline $9 c$ & 85.88 & 67.02 & 4 & 254.01 & 281.31 & 3.04 & 5 & 2 & 0 \\
\hline 9d & 89.06 & 57.78 & 3 & 242.00 & 285.73 & 3.66 & 4 & 2 & 0 \\
\hline $9 e$ & 89.06 & 57.78 & 3 & 233.39 & 269.28 & 3.15 & 4 & 2 & 0 \\
\hline 9f & 89.06 & 57.78 & 3 & 245.02 & 265.32 & 3.43 & 4 & 2 & 0 \\
\hline $9 \mathrm{~g}$ & 89.06 & 57.78 & 2 & 211.66 & 237.26 & 3.30 & 4 & 2 & 0 \\
\hline $9 h$ & 73.25 & 103.61 & 3 & 234.99 & 282.26 & 3.21 & 7 & 2 & 0 \\
\hline $9 i$ & 73.25 & 103.61 & 3 & 234.99 & 282.26 & 3.26 & 7 & 2 & 0 \\
\hline 9j & 73.25 & 103.61 & 3 & 234.99 & 282.26 & 3.23 & 7 & 2 & 0 \\
\hline 9k & 89.06 & 57.78 & 2 & 229.54 & 316.16 & 4.11 & 4 & 2 & 0 \\
\hline 91 & 89.06 & 57.78 & 2 & 238.73 & 306.15 & 4.58 & 4 & 2 & 0 \\
\hline $9 \mathrm{~m}$ & 89.06 & 57.78 & 2 & 216.59 & 255.25 & 3.46 & 4 & 2 & 0 \\
\hline 9n & 89.06 & 57.78 & 2 & 225.19 & 271.71 & 3.98 & 4 & 2 & 0 \\
\hline 90 & 89.06 & 57.78 & 2 & 225.19 & 271.71 & 3.95 & 4 & 2 & 0 \\
\hline Erlotinib & 83.31 & 74.45 & 10 & 362.06 & 393.44 & 2.79 & 7 & 1 & 0 \\
\hline
\end{tabular}

Abbreviations: ABS\%, percentage absorption; TPSA, topological polar surface area; $\mathrm{n}$-ROTB, number of rotatable bonds; $\mathrm{MV}$, molecular volume; $\mathrm{MW}$, molecular weight; miLog P, logarithm of partition coefficient of the compound between n-octanol and water; n-ON acceptors, number of hydrogen bond acceptors; n-OHNH donors, number of hydrogen bonds donors. 
taching a phenoxy group at the 6-position could be beneficial for the activity due to the proper disposition of the compound into the hydrophobic pocket of the enzyme. Based on ADME prediction analysis, the synthesized compounds possessed excellent druglikeness properties to be formulated for the oral route of administration. The extension of this work would provide valuable facts for the further design of EGFR-TKIs.

\section{Acknowledgments}

This work was supported by a grant from the Research Council of Shahid Beheshti University of Medical Sciences (No. 18750) and the National Institute for Medical Research Development (No. 962440).

\section{References}

1. Fayed EA, Eissa SI, Bayoumi AH, Gohar NA, Mehany ABM, Ammar YA. Design, synthesis, cytotoxicity and molecular modeling studies of some novel fluorinated pyrazole-based heterocycles as anticancer and apoptosis-inducing agents. Mol Divers. 2019;23(1):165-81. doi: 10.1007/s11030-018-9865-9. [PubMed: 30099687].

2. Nepali K, Sharma S, Sharma M, Bedi PM, Dhar KL. Rational approaches, design strategies, structure activity relationship and mechanistic insights for anticancer hybrids. Eur J Med Chem. 2014;77:422-87. doi: 10.1016/j.ejmech.2014.03.018. [PubMed: 24685980].

3. Elmetwally SA, Saied KF, Eissa IH, Elkaeed EB. Design, synthesis and anticancer evaluation of thieno[2,3-d]pyrimidine derivatives as dual EGFR/HER2 inhibitors and apoptosis inducers. Bioorg Chem. 2019;88:102944. doi: 10.1016/j.bioorg.2019.102944. [PubMed: 31051400].

4. Du Z, Lovly CM. Mechanisms of receptor tyrosine kinase activation in cancer. Mol Cancer. 2018;17(1):58. doi: 10.1186/s12943-018-0782-4. [PubMed: 29455648]. [PubMed Central: PMC5817791].

5. Eldehna WM, Abo-Ashour MF, Nocentini A, Gratteri P, Eissa IH, Fares $\mathrm{M}$, et al. Novel 4/3-((4-oxo-5-(2-oxoindolin-3-ylidene)thiazolidin2-ylidene)amino) benzenesulfonamides: Synthesis, carbonic anhydrase inhibitory activity, anticancer activity and molecular modelling studies. Eur J Med Chem. 2017;139:250-62. doi: 10.1016/j.ejmech.2017.07.073. [PubMed: 28802125].

6. Schlessinger J. Cell signaling by receptor tyrosine kinases. Cell. 2000;103(2):211-25. doi: 10.1016/s0092-8674(00)00114-8. [PubMed: 11057895].

7. McDonell LM, Kernohan KD, Boycott KM, Sawyer SL. Receptor tyrosine kinase mutations in developmental syndromes and cancer: two sides of the same coin. Hum Mol Genet. 2015;24(R1):R606. doi: 10.1093/hmg/ddv254. [PubMed: 26152202]. [PubMed Central: PMC4572000].

8. Ferguson FM, Gray NS. Kinase inhibitors: the road ahead. Nat Rev Drug Discov. 2018;17(5):353-77. doi: 10.1038/nrd.2018.21. [PubMed: 29545548].

9. Qiu KM, Wang HH, Wang LM, Luo Y, Yang XH, Wang XM, et al. Design, synthesis and biological evaluation of pyrazolyl-thiazolinone derivatives as potential EGFR and HER-2 kinase inhibitors. Bioorg Med Chem. 2012;20(6):2010-8. doi: 10.1016/j.bmc.2012.01.051. [PubMed: 22361272].

10. Herbst RS, Morgensztern D, Boshoff C. The biology and management of non-small cell lung cancer. Nature. 2018;553(7689):446-54. doi: 10.1038/nature25183. [PubMed: 29364287].
11. Blume-Jensen $\mathrm{P}$, Hunter T. Oncogenic kinase signalling. Nature 2001;411(6835):355-65. doi: 10.1038/35077225. [PubMed: 11357143].

12. Kim H, Muller WJ. The role of the epidermal growth factor receptor family in mammary tumorigenesis and metastasis. Exp Cell Res. 1999;253(1):78-87. doi: 10.1006/excr.1999.4706. [PubMed:10579913].

13. Webb JD, Simon MC. Novel insights into the molecular origins and treatment of lung cancer. Cell Cycle. 2010;9(20):4098-105. doi: 10.4161/cc.9.20.13588. [PubMed: 20962595]. [PubMed Central: PMC3055194].

14. Brambilla E, Travis WD, Colby TV, Corrin B, Shimosato Y. The new World Health Organization classification of lung tumours. Eur Respir J. 2001;18(6):1059-68. doi: 10.1183/09031936.01.00275301. [PubMed: 11829087].

15. Qin X, Li Z, Yang L, Liu P, Hu L, Zeng C, et al. Discovery of new $[1,4]$ dioxino[2,3-f]quinazoline-based inhibitors of EGFR including the T790M/L858R mutant. Bioorg Med Chem. 2016;24(13):2871-81. doi: 10.1016/j.bmc.2016.01.003. [PubMed: 27234887].

16. Tabatabai S, Nematpour M, Rezaee E, Jahani M. A Copper-Catalyzed Synthesis of Functionalized Quinazolines from Isocyanides and Aniline Tri- and Dichloroacetonitrile Adducts through Intramolecular C-H Activation. Synlett. 2017;28(12):1441-4. doi: 10.1055/s-003615588166.

17. Tu Y, Wang C, Yang Z, Zhao B, Lai L, Yang Q, et al. Discovery of novel quinazoline derivatives bearing semicarbazone moiety as potent EGFR kinase inhibitors. Comput Struct Biotechnol J. 2018;16:462-78. doi: 10.1016/j.csbj.2018.10.016. [PubMed: 30455856]. [PubMed Central: PMC6232652].

18. Das D, Xie L, Wang J, Xu X, Zhang Z, Shi J, et al. Discovery of new quinazoline derivatives as irreversible dual EGFR/HER2 inhibitors and their anticancer activities - Part 1. Bioorg Med Chem Lett. 2019;29(4):591-6. doi: 10.1016/j.bmcl.2018.12.056. [PubMed: 30600209].

19. OuYang Y, Zou W, Peng L, Yang Z, Tang Q, Chen M, et al. Design, synthesis, antiproliferative activity and docking studies of quinazoline derivatives bearing 2,3-dihydro-indole or 1,2,3,4-tetrahydroquinoline as potential EGFR inhibitors. Eur J Med Chem. 2018;154:29-43. doi: 10.1016/j.ejmech.2018.05.006. [PubMed: 29775935].

20. Zhang Y, Gao H, Liu R, Liu J, Chen L, Li X, et al. Quinazoline-1deoxynojirimycin hybrids as high active dual inhibitors of EGFR and alpha-glucosidase. Bioorg Med Chem Lett. 2017;27(18):4309-13. doi: 10.1016/j.bmcl.2017.08.035. [PubMed: 28838691].

21. Eberlein CA, Stetson D, Markovets AA, Al-Kadhimi KJ, Lai Z, Fisher $\mathrm{PR}$, et al. Acquired Resistance to the Mutant-Selective EGFR Inhibitor AZD9291 Is Associated with Increased Dependence on RAS Signaling in Preclinical Models. Cancer Res. 2015;75(12):2489-500. doi: 10.1158/0008-5472.CAN-14-3167. [PubMed: 25870145]. [PubMed Central: PMC4605607].

22. Zhang $\mathrm{Y}$, Chen L, Xu H, Li X, Zhao L, Wang W, et al. 6,7 Dimorpholinoalkoxy quinazoline derivatives as potent EGFR inhibitors with enhanced antiproliferative activities against tumor cells. EurJMed Chem. 2018;147:77-89. doi:10.1016/j.ejmech.2018.01.090. [PubMed: 29421573].

23. Lipinski CA, Lombardo F, Dominy BW, Feeney PJ. Experimental and computational approaches to estimate solubility and permeability in drug discovery and development settings. Adv Drug Deliv Rev. 2001;46(1-3):3-26. doi: 10.1016/s0169-409x(00)00129-0. [PubMed: 11259830].

24. Zhao YH, Abraham MH, Le J, Hersey A, Luscombe CN, Beck G, et al. Rate-limited steps of human oral absorption and QSAR studies. Pharm Res. 2002;19(10):1446-57. doi: 10.1023/a:1020444330011. [PubMed: 12425461].

25. Clark RH, Wagner EC. Isatoic Anhydride. I. Reactions with Primary and Secondary Amines and with Some Amides1. J Org Chem. 2002;9(1):5567. doi: 10.1021/jo01183a007. 
26. Ertl P, Rohde B, Selzer P. Fast calculation of molecular polar surface area as a sum of fragment-based contributions and its appli- cation to the prediction of drug transport properties. J Med Chem. 2000;43(20):3714-7. doi: 10.1021/jm000942e. [PubMed: 11020286]. 